\title{
Numerical modeling of the electron beam welding and its experimental validation
}

\author{
M. Chiumenti ${ }^{\text {a,* }}$, M. Cervera ${ }^{a}$, N. Dialami ${ }^{a}$, B. Wu ${ }^{b}$, L. Jinwei ${ }^{b}$, C. Agelet de Saracibar ${ }^{a}$ \\ ${ }^{a}$ International Center for Numerical Methods in Engineering (CIMNE), Universidad Politécnica de Cataluña (UPC), Edificio C1, Campus Norte, \\ Gran Capitán s/n, 08034 Barcelona, Spain \\ ${ }^{\mathrm{b}}$ Aviation Industry Corporation of China (AVIC), Beijing Aeronautical Manufacturing Technology Research Institute (BAMTRI), Dongjunzhuang Road, \\ Beijing 100024, China
}

\section{A R T I C L E I N F O}

\section{Article history:}

Received 1 December 2015

Received in revised form

22 June 2016

Accepted 7 July 2016

\section{Keywords:}

Electron Beam Welding (EBW)

Thermo-mechanical

Phase-change

Plasticity

\begin{abstract}
A B S T R A C T
Electron Beam Welding (EBW) is a highly efficient and precise welding method increasingly used within the manufacturing chain and of growing importance in different industrial environments such as the aeronautical and aerospace sectors. This is because, compared to other welding processes, EBW induces lower distortions and residual stresses due to the lower and more focused heat input along the welding line.

This work describes the formulation adopted for the numerical simulation of the EBW process as well as the experimental work carried out to calibrate and validate it.

The numerical simulation of EBW involves the interaction of thermal, mechanical and metallurgical phenomena. For this reason, in this work the numerical framework couples the heat transfer process to the stress analysis to maximize accuracy. An in-house multi-physics FE software is used to deal with the numerical simulation. The definition of an ad hoc moving heat source is proposed to simulate the EB power surface distribution and the corresponding absorption within the work-piece thickness. Both heat conduction and heat radiation models are considered to dissipate the heat through the boundaries of the component. The material behavior is characterized by an apropos thermo-elasto-viscoplastic constitutive model. Titanium-alloy Ti6A14V is the target material of this work.

From the experimental side, the EB welding machine, the vacuum chamber characteristics and the corresponding operative setting are detailed. Finally, the available facilities to record the temperature evolution at different thermo-couple locations as well as to measure both distortions and residual stresses are described. Numerical results are compared with the experimental evidence.
\end{abstract}

(c) 2016 Elsevier B.V. All rights reserved.

\section{Introduction}

Electron Beam Welding (EBW) is a fusion welding process in which a beam of high velocity electrons is applied to the materials being joined. The work-pieces melt as the kinetic energy of the electrons is transformed into heat upon impact.

In an electron beam system the electron emission originates from a negatively charged filament: the cathode. When heated up to its thermionic emission temperature, this filament emits electrons. These electrons are accelerated by the electric field between a negatively charged bias electrode (located slightly below the cathode) and the anode. They pass through the hole in the anode and are focused by an electromagnetic coil to a point at the work-

\footnotetext{
* Corresponding author.

E-mail address: michele@cimne.upc.edu (M. Chiumenti).

URL: http://www.cimne.com (M. Chiumenti).
}

piece surface (see Fig. 1a). The beam currents and the accelerating voltages employed for typical EBW vary over the ranges of 50$1000 \mathrm{~mA}$ and $30-175 \mathrm{~kW}$, respectively.

Typically, the EBW system used in manufacturing operates in a vacuum environment providing protection against oxidation and removing gas molecules to guarantee a stable electron beam emission. Moreover, electrons are scattered when they hit air molecules so that the lower is the ambient pressure, the less is the scattering. As a consequence, the beam diameter reduces by decreasing the ambient pressure and its power can be focused in a very small spot area in the range of $0.3-0.8 \mathrm{~mm}$ and the resulting power density can be as high as $1000 \mathrm{~W} / \mathrm{m}^{2}$.

The resulting weld is very narrow and the high energy density allows for quick travel speeds. Therefore, the welding process occurs so fast that the adjacent material does not absorb the exceeding heat, leading to a minimal heat affected zone.

On the one hand, in the case of thin plates, the welding process is induced by the material melting within the welding pool 
a

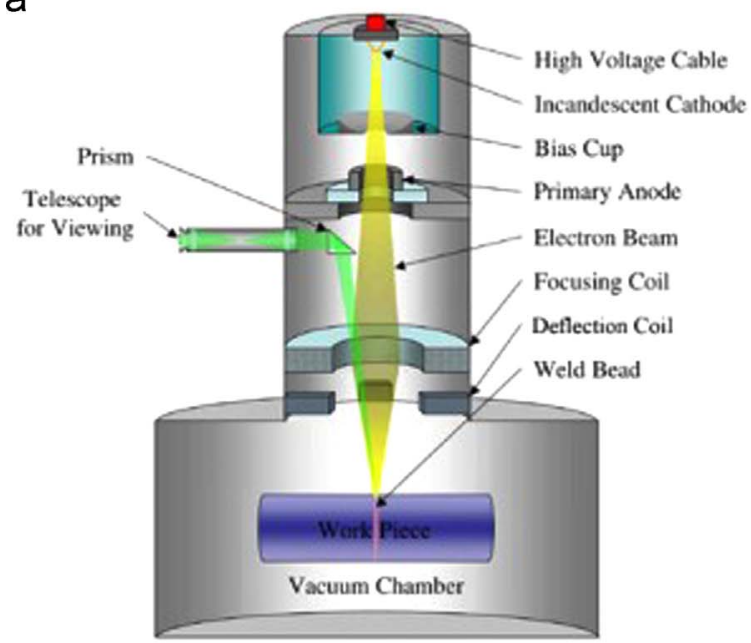

EB welding machine

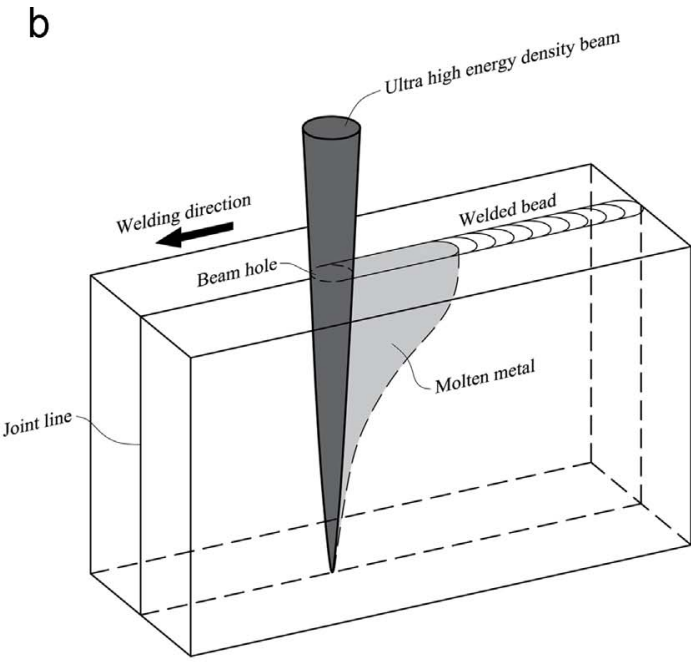

Molten pool

Fig. 1. EBW process. Images courtesy of: Joining Technologies, Inc.

throughout the thickness of the joint. On the other hand, when a deeper penetration is required, the high power density of the EB leads to the vaporization of the material, producing the, so-called, keyhole throughout the thickness of the specimen (see Fig. 1b). The material flow in the molten-pool around the electron beam closes the gap of the welding joint and the following coalescence process produces the fusion weld.

Numerical modeling of welding processes has proven to be highly efficient for design and production engineering [25,33,27$29,16]$. In comparison with experimental studies, numerical simulation can give detailed information concerning the characteristics of the welding pool, the size of the heat affected zone and the final distortions and the induced residual stresses $[17,18,30]$ and, particularly, their relationship with the welding process parameters such as the welding speed, the EB power, and the spot size $[26,34]$. Hence, the experimental work can be reduced as well as the trial-and-error loop necessary to optimize the final manufacturing process.

Sections 2-5 describe the FE framework for the thermomechanical analysis of the EBW process including a detailed description of the power source induced by the EB as well as the constitutive model suitable for the entire temperature range, from room temperature to the fusion temperature of the material. In Section 6, the research equipment available at the Beijing Aeronautical Manufacturing Technology Research Institute (BAMTRI) is presented. The experimental campaign carried out to calibrate the numerical model is illustrated in Section 7. Also, the simulation tool is assessed by analyzing an industrial component, part of the AIRBUS door frame.

\section{Heat transfer analysis}

Both the solidification and the cooling phases are controlled by the balance of energy equation. This governing equation can be stated as:

$\dot{H}=-\nabla \cdot \mathbf{q}+\dot{Q}+\dot{D}_{\text {mech }}$

where $\dot{H}$ is the enthalpy rate (per unit of volume), $\mathbf{q}$ is the heat flux, while $\dot{Q}$ and $\dot{D}_{\text {mech }}$ represent the heat source (per unit of volume) and the thermo-mechanical dissipation rate (per unit of volume), respectively.
For the welding process, the heat source is the power input of the EB through the thickness of the component. A detailed description of this heat source is presented in the following section. The thermo-mechanical dissipation can be neglected in front of the power source of the electron beam.

The enthalpy $H\left(T, f_{L}\right)$ is a state variable defined as a function of the temperature, $T$, and the liquid fraction, $f_{L}$. Hence, the enthalpy rate in (1) results in:

$\dot{H}\left(T, f_{L}\right)=\frac{\partial H_{\dot{H}}}{\partial T} \dot{T}+\frac{\partial H_{f^{\prime}}}{\partial f_{L}}=C \dot{T}+L \dot{f}_{L}$

where $C(T)=\frac{\partial H}{\partial T}$ is the (temperature dependent) heat capacity and $L=\frac{\partial H}{\partial f_{L}}$ is the latent heat released during the phase-change process.

The heat capacity is usually defined as: $C=\rho c$, the product of the material density, $\rho$, and the specific heat, $c$.

During the phase transformation the material volume, $V$, can be split into liquid and solid phases as: $V=V_{L}+V_{S}$. The liquid and solid fractions are defined as: $f_{L}=\frac{V_{L}}{V}$ and $f_{S}=\frac{V_{S}}{V}$, respectively, so that: $f_{L}+f_{S}=1$. The evolution of the liquid fraction $\dot{f}_{L}$ or, alternatively, $\dot{S}_{S}=-\dot{f}_{L}$ defines the phase-change, that is, how the latent heat is absorbed or released during the transformation.

Remark 1. On the one hand, the EBW induces the melting of the material in the HAZ followed by the solidification process which produces the coalescence of the joint. On the other hand, the energy balance (accounting for the energy absorbed during the melting and the energy released during the solidification) is null and very localized in the HAZ.

Remark 2. The amount of latent heat is negligible in front of the energy input coming from the EBW. Moreover, the Heat Affected Zone (HAZ) is very narrow and the phase-change occurs very fast compared to the thermal diffusion process, so that its global effect is minor in the heat transfer analysis (see [14]).

The heat flux (per unit of surface) $\mathbf{q}$ is computed as a function of the temperature gradient through the law of Fourier as:

$\mathbf{q}=-k \nabla T$

where $k(T)$ is the (temperature dependent) thermal conductivity. Due to the high conductivity of the metallic materials, the thermal diffusion process is the key mechanism to form the welding joint, driving both the solidification and the cooling phases through the thickness of the body and particularly in the molten-pool. 


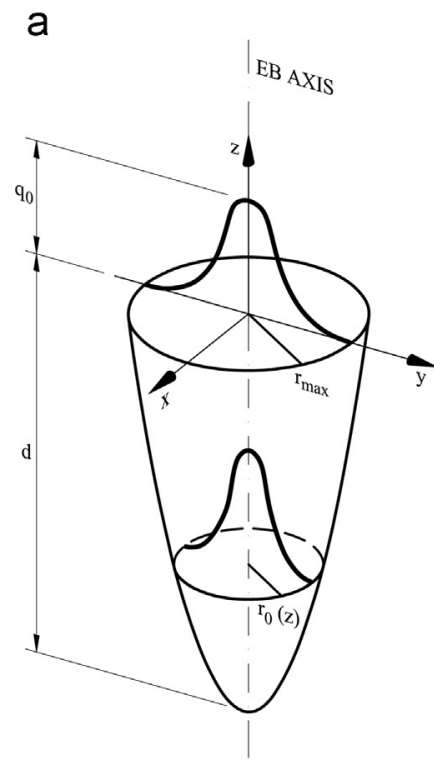

Power density distribution function b

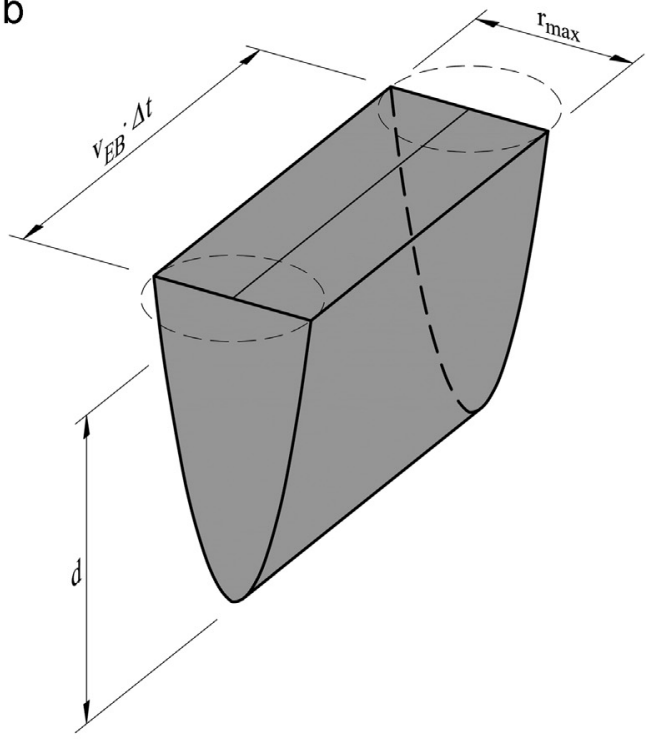

Power distribution used in the FE analysis

Fig. 2. EBW process. Power source.

Let $V$ be an open and bounded domain in $\mathbb{R}^{n_{\text {dim }}}$ where $n_{\text {dim }}$ is the number of dimensions of the space, closed by the smooth boundary $S=S_{T} \cup S_{q}$ where the corresponding boundary conditions are defined in terms of either prescribed temperature on $S_{T}$ or prescribed heat flux on $S_{q}$. Suitable initial conditions for the transient thermal problem are defined in terms of initial temperature field: $T(t=0)=T_{0}$.

The resulting weak (integral) form of the energy balance equation (1), used for the heat transfer analysis, can be written as:

$\int_{V}\left[\left(\rho c \dot{T}+L \dot{f}_{L}\right) \delta T\right] d V+\int_{V}[k \nabla T \cdot \nabla(\delta T)] d V=W_{\text {ther }}^{\text {ext }} \quad \forall \delta T$

where $\delta T$ are the variations of the temperature field, compatible with the Dirichlet boundary conditions (test functions), and $W_{\text {ther }}^{\text {ext }}$ denotes the external work of the thermal loads:

$$
\begin{aligned}
W_{\text {ther }}^{\text {ext }}(\delta T)= & \int_{V}\left[\left(\dot{D}_{\text {mech }}+\dot{Q}\right) \delta T\right] d V \\
& -\int_{S_{q}}\left[\left(\bar{q}+q_{\text {cond }}+q_{\text {conv }}+q_{\text {rad }}\right) \delta T\right] d S
\end{aligned}
$$

If the welding process is used to join small thickness samples, the prescribed heat flux, $\bar{q}$, defined in (5), can be used to apply the EB power input on the welding joint surface.

Taking into account that the EBW process is (usually) performed into a vacuum chamber (around $10^{-6}$ bar), the heat flux by convection, $q_{\text {conv }}$, can be neglected. Hence, the radiation heat flux, $q_{\text {rad }}$, is the most important condition to dissipate the heat through the body surfaces and particularly at the HAZ, due to the high temperature field induced by the welding heat source. The radiation heat flux can be computed using Stefan-Boltzmann's law as a function of the surface temperature, $T$, and the room temperature, $T_{\text {env }}$, as:

$q_{\text {rad }}=\sigma_{\text {rad }} \varepsilon_{\text {rad }}\left(T^{4}-T_{\text {env }}^{4}\right)$

where $\sigma_{\text {rad }}$ is the Stefan-Boltzmann constant and $\varepsilon_{\text {rad }}$ is the emissivity parameter, respectively.

Remark 3. The radiation heat flux out of the HAZ can be neglected. This means that its global effect could be considered by modifying the efficiency parameter of the heat source (see next section) without loss of accuracy in the simulation.

Finally, the heat loss due to the heat conduction process between the component and the clamping system, $q_{\text {cond }}$, can be taken into account using Newton's law as:

$q_{\text {cond }}=h_{\text {cond }}\left(T-T_{\text {clamp }}\right)$

where $h_{\text {cond }}$ is the heat transfer coefficient (HTC) by conduction between the two materials in contact, and $T_{\text {clamp }}$ is the reference temperature of the clamping system (see [11]).

Remark 4. The HTC is defined as the inverse of the thermal resistivity and it depends on different parameters defined at the contact interface such as the contact pressure and the surface roughness, among others. Considering the contact between metallic materials, the value of the HTC is very high, around 2000$3000 \mathrm{~W} / \mathrm{m}^{2} \mathrm{~K}$. Hence, Newton's law can be replaced by prescribing the temperature at the corresponding contact surface, $S_{T}$ as: $T=T_{\text {clamp }}$.

\section{EB power source}

The heat source per unit of volume of the EB, appearing in the balance of energy equation (1), is defined as:

$\dot{Q}=\dot{P} q(r, z)$

where $\dot{P}$ is total energy input and $q(r, z)$ is the distribution function depending on the distance, $r$, from the central axis of the EB and the penetration, $z$, through the thickness of the work piece.

The power source is:

$\dot{P}=\eta V I$

where $V$ and $I$ are the accelerating voltage and the current intensity, respectively, and $\eta$ is the heat absorption efficiency.

There exist different alternatives for the definition of the power distribution $q(r, z)[33,21,31,25,23,32,21]$. In this work, the EB is characterized by a Gaussian distribution defined as:

$q(r, z)=q_{o} e^{-3\left[\frac{r}{r_{0}(z)}\right]^{2}} \quad 0 \leq r \leq r_{o}(z) ; \quad-d \leq z \leq 0$ 
$r_{o}(z)=r_{\max } \sqrt{\frac{z+d}{d}}$ being the radius of the circular spot area, where the $95 \%$ of the energy is focused. This radius is maximum at the top surface where $r_{o}(0)=r_{\max }$, and it reduces, according to a parabolic function, till vanishing at the maximum penetration $d$, of the EB through the weld joint (see Fig. 2a).

The proposed distribution of the power source is normalized (its volume integral is 1) and its amplitude is maintained for each section along the beam axis as:

$q_{o}=\frac{2}{d r_{\max }^{2}}$

\section{FE modeling of the EBW process}

The EBW process is modeled by applying the (ideal) power distribution defined in (8) for a heat source which is moving along the user-defined welding line. From the numerical point of view, the implications derived from the space discretization (FE mesh adopted) and the time-stepping defined for the time-integration of the thermal (transient) problem must be considered.

The numerical simulation of this process requires an ad hoc procedure to apply the (volumetric) heat source to those elements affected by the EB while moving. Therefore, at each time-step of the simulation, a searching algorithm is used to identify the elements within the HAZ.

The discrete problem is characterized by a time-step, $\Delta t=t^{n+1}-t^{n}$, so that a discrete sequence of computations is performed according to the time-marching scheme. Therefore, the heat source does not continuously move along the welding line (as for the continuous problem) but, instead, it is stepping from its position at time $t^{n}$ to that at time $t^{n+1}$. Consequently, the power input is intermittent along the welding line, producing a discontinuous HAZ. The problem can be alleviated by reducing the time-step, to get an overlapped HAZ progression, but not avoided.

As a possible alternative, the total power input delivered within the time-step, $\Delta t$, can be distributed to the elements belonging to the volume affected by the EB during this interval, which has at both ends the molten-pools at times $t^{n}$ and $t^{n+1}$. The upper surface of the HAZ results in a rectangular section $v_{E B} \Delta t$ long ( $v_{E B}$ being the EBW speed) and $r_{\max }$ wide (see Fig. 2b). The section width reduces in depth according to the parabolic evolution described above (see [14]).

A further consideration is about the space discretization. Even if the heat affected zone (HAZ) is discretized using a very fine mesh, it is very challenging to have enough resolution to define exactly

\section{a}

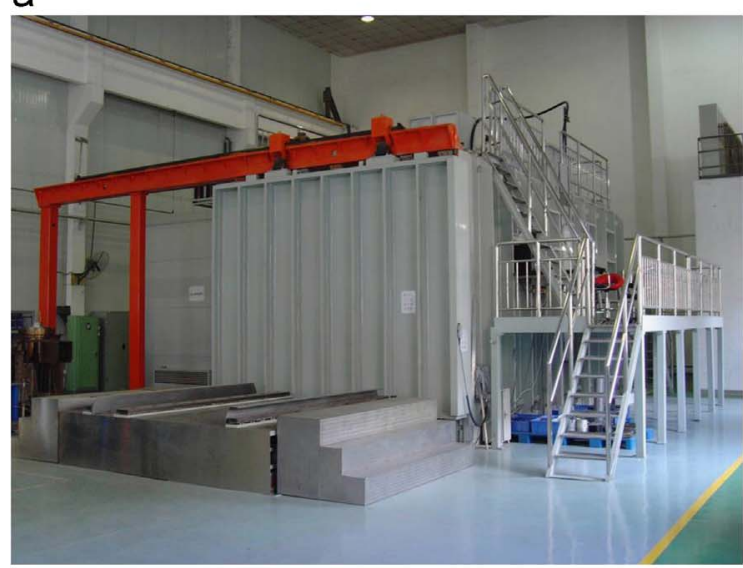

High vacuum EBW equipment the ideal volume, $V^{H A Z}$, where the power input is applied. According to the outcome of the searching algorithm, it is possible to compute the volume, $V_{h}{ }^{H A Z}$, as the sum of all the volumes, $V^{(e)}$, of the elements belonging to the (discretized) HAZ as:

$V_{h}^{H A Z}=\left.\sum_{e=1}^{n e} V^{(e)}\right|_{e \in H A Z}$

Hence, the (volumetric) heat source is corrected as:

$\dot{P}_{h}=\frac{V^{H A Z}}{V_{h}^{H A Z}} \dot{P}$

This correction preserves the total energy input independently of the mesh used (see [12]).

\section{Stress analysis}

The mechanical problem is governed by the balance of momentum equation. The local form, for quasi-static conditions, can be stated as: find the displacement field, $\mathbf{u}$, for given (prescribed) body forces $\mathbf{b}$, such that:

$\nabla \cdot \boldsymbol{\sigma}(\mathbf{u})+\mathbf{b}=\mathbf{0}$

where $\nabla \cdot(\cdot)$ is the divergence operator and $\boldsymbol{\sigma}(\mathbf{u})$ denotes the Cauchy stress tensor.

The stress tensor can be split into its hydrostatic (pressure) and deviatoric parts $p$ and $\mathbf{s}$, respectively, as:

$\boldsymbol{\sigma}(\mathbf{u}, p)=p \mathbf{I}+\mathbf{S}(\mathbf{u})$

being $p=\frac{1}{3} \operatorname{tr}(\boldsymbol{\sigma})$ and $\mathbf{s}=\operatorname{dev}(\boldsymbol{\sigma})$. This is a very convenient choice to deal with the isochoric behavior, which is a characteristic of the liquid phase or, more generally, when the deformations are mainly deviatoric. This is the case when J2-plasticity models are used to describe the constitutive behavior of metallic alloys.

This given, the local form of the mechanical problem can be reformulated as: find the displacement field, $\mathbf{u}$, and the pressure field, $p$ (defined as an independent variable of the problem), such that:

$\nabla \cdot \mathbf{s}(\mathbf{u})+\nabla p+\mathbf{b}=\mathbf{0}$

$\left(\nabla \cdot \mathbf{u}-e^{T}\right)-\frac{p}{K}=0$

where $e^{T}$ is the thermal deformation and $K(T)$ is the (temperature dependent) bulk modulus which controls the material compressibility. Note that the mixed $\mathbf{u} / p$ formulation in (16)-(17) is equivalent to the balance of momentum equation (14) but, in this split format, it

b

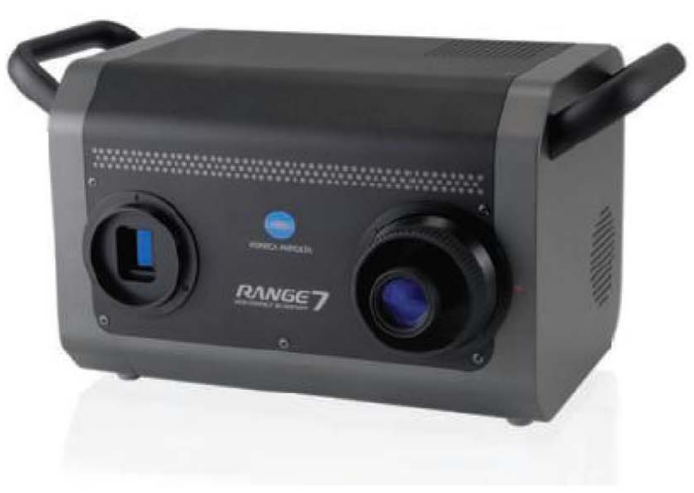

3D scanner

Fig. 3. EB welding facilities at BAMTRI research laboratories. 
is suitable for both the compressible and incompressible cases. In particular, when the material is liquid, the bulk modulus $K \rightarrow \infty$, so that Eq. (17) enforces the volumetric constraint as:

$\nabla \cdot \mathbf{u}=e^{T}$

Eqs. (16) and (17) must be integrated over the volume of the domain, $V$, occupied by the solid. Let $S$ be the corresponding boundary, split into $S_{\sigma}$ and $S_{u}$, being $S=S_{\sigma} \cup S_{u}$, such that tractions are prescribed on $S_{\sigma}$, while displacements are specified on $S_{u}$, respectively.

The weak (integral) form of the mechanical problem defined by the mixed $\mathbf{u} / p$ formulation in (16) and (17) is:

$$
\begin{cases}\int_{V}[(\nabla \cdot \mathbf{s}(\mathbf{u})) \cdot \delta \mathbf{v}] d V+\int_{V}(\nabla p \cdot \delta \mathbf{v}) d V+\int_{V}(\mathbf{b} \cdot \delta \mathbf{v}) d V=0 & \forall \delta \mathbf{v} \\ \int_{V}\left[\left(\nabla \cdot \mathbf{u}-e^{T}-\frac{p}{K}\right) \delta p\right] d V=0 & \forall \delta p\end{cases}
$$

where $\delta \mathbf{v}$ and $\delta p$ are the variations of displacements and pressure fields, respectively, compatible with the Dirichlet boundary conditions (test functions).

Integrating by parts, the following expressions are obtained:

$$
\begin{aligned}
\int_{V}[(\nabla \cdot \mathbf{s}(\mathbf{u})) \cdot \delta \mathbf{v}] d V & +\int_{V}(\nabla p \cdot \delta \mathbf{v}) d V=-\int_{V}\left(\mathbf{s}(\mathbf{u}): \nabla^{S} \delta \mathbf{v}\right) d V \\
& -\int_{V}(p \nabla \cdot \delta \mathbf{v}) d V+\int_{S_{\sigma}}(\overline{\mathbf{t}} \cdot \delta \mathbf{v}) d S
\end{aligned}
$$

where $\overline{\mathbf{t}}$ are prescribed tractions on $S_{\sigma}$.

Substituting (20) into (19), the mixed $\mathbf{u} / p$ variational form of the quasi-static mechanical problem yields:

$$
\begin{cases}\int_{V}\left(\mathbf{s}(\mathbf{u}): \nabla^{s} \delta \mathbf{v}\right) d V+\int_{V}(p \nabla \cdot \delta \mathbf{v}) d V=W_{\text {mech }}^{\text {ext }} & \forall \delta \mathbf{u} \\ \int_{V}\left[\left(\nabla \cdot \mathbf{u}-e^{T}-\frac{p}{K}\right) \delta p\right] d V=0 & \forall \delta p\end{cases}
$$

where $W_{\text {mech }}^{\text {ext }}$ denotes the external work of the mechanical loads, defined as:

$W_{m e c h}^{\text {ext }}(\delta \mathbf{v})=\int_{\Omega_{\chi}}(\mathbf{b} \cdot \delta \mathbf{v}) d V+\int_{\partial \Omega_{\sigma}}(\overline{\mathbf{t}} \cdot \delta \mathbf{v}) d S$

\subsection{Mechanical constitutive laws}

The material model chosen to simulate the constitutive behavior is based on previous developments of the authors in the field of coupled thermo-mechanical analysis for casting processes $[1,6]$ or welding analysis [12]. In both cases, the temperature range varies from room temperature to very high values above the melting point. As a consequence, the material response must reproduce either the elasto-plastic behavior when the material is at room temperature or the pure viscous behavior observed above the melting point. This transition can be simulated adopting an apropos thermo-elasto-visco-plastic constitutive model. The definition of temperature dependent material properties and, particularly, the thermal softening induced by the temperature dependent yield stress, allows for a gradual contraction of the von Mises yield-surface as the temperature increases. When the temperature is close to the melting point, the viscous behavior becomes predominant, the elastic limit gradually reduces, vanishing when liquidus temperature is reached. As a result, a purely viscous model is recovered for the liquid-like behavior. Hence, the transition from the solid to the liquid-like phase is driven by the temperature dependency of the material properties and it is forced during the phase-change according to the solid fraction function.

Therefore, the constitutive laws suitable for all the temperature range of the process are characterized by a J2-thermo-elastovisco-plastic constitutive model of the form:

$p=\frac{K}{f_{S}}\left(e^{v o l}-e^{T}\right)$

$\mathbf{s}=\frac{2 G}{f_{S}}\left(\mathbf{e}-\mathbf{e}^{v p}\right)$

where $G(T)$ is the (temperature dependent) shear modulus and $K(T)$ is the (temperature dependent) bulk modulus which controls the material compressibility.

Remark 5. During the solid-to-liquid phase-change, the solid fraction, $f_{S} \rightarrow 0$, while the stresses remain bounded. As a consequence, both the volumetric and the deviatoric parts of the elastic deformation vanish and the material behaves as a liquidlike material. The (deviatoric) viscous-plastic flow is obtained from the rate-dependent visco-plastic strain evolution law.

The total strain tensor is computed in terms of the displacement field, u, as:

$\boldsymbol{\varepsilon}(\mathbf{u})=\nabla^{s} \mathbf{u}$

and its volumetric and deviatoric parts are obtained as:

$e^{v o l}=\operatorname{tr}(\boldsymbol{\varepsilon})=\nabla \cdot \mathbf{u}$

$\mathbf{e}=\operatorname{dev}(\boldsymbol{\varepsilon})=\boldsymbol{\varepsilon}-\left(\frac{e^{v o l}}{3}\right) \mathbf{I}$

The thermal deformation $e^{T}(T)$ is defined as a volumetric term of the form:

$e^{T}(T)=e^{c o o l}(T)+e^{p c}(T)$ a

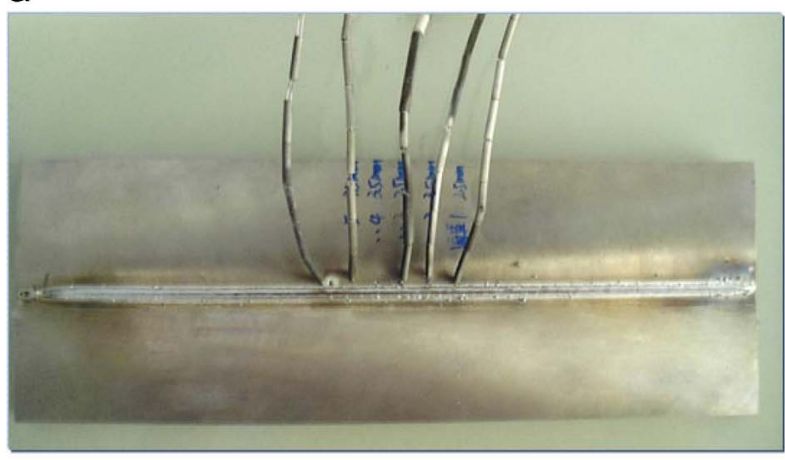

Thermocouples location b

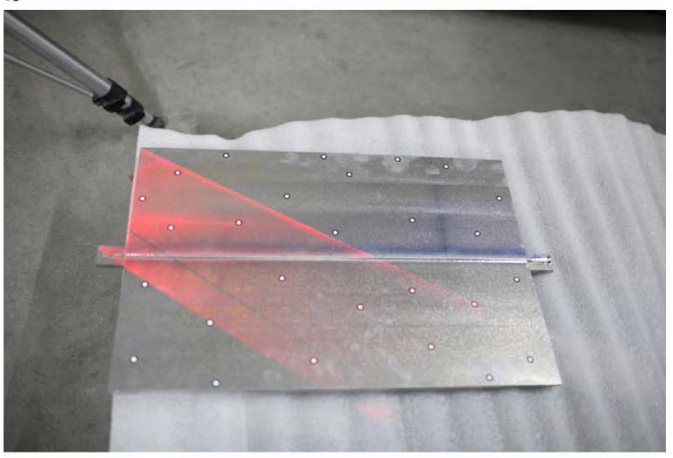

Scanning operation

Fig. 4. Experimental settings for temperature and distortions measurements. 


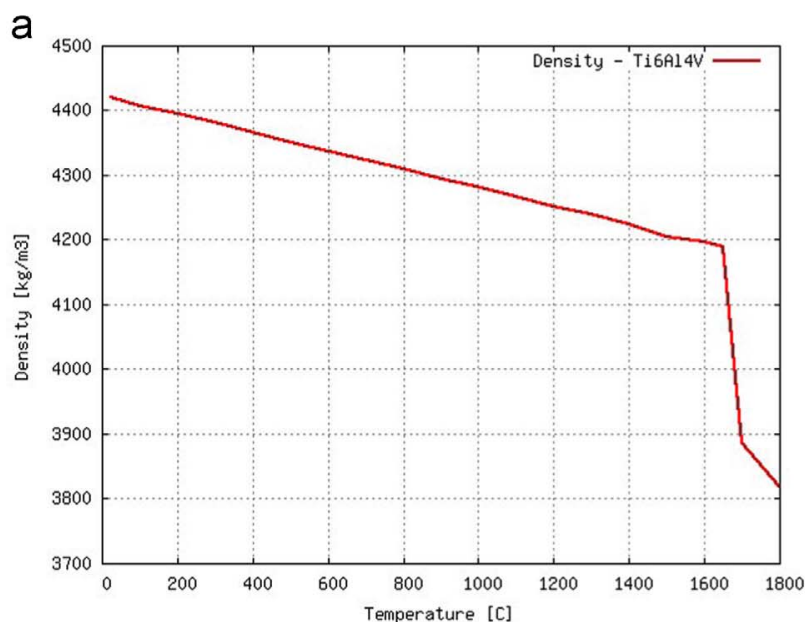

Density

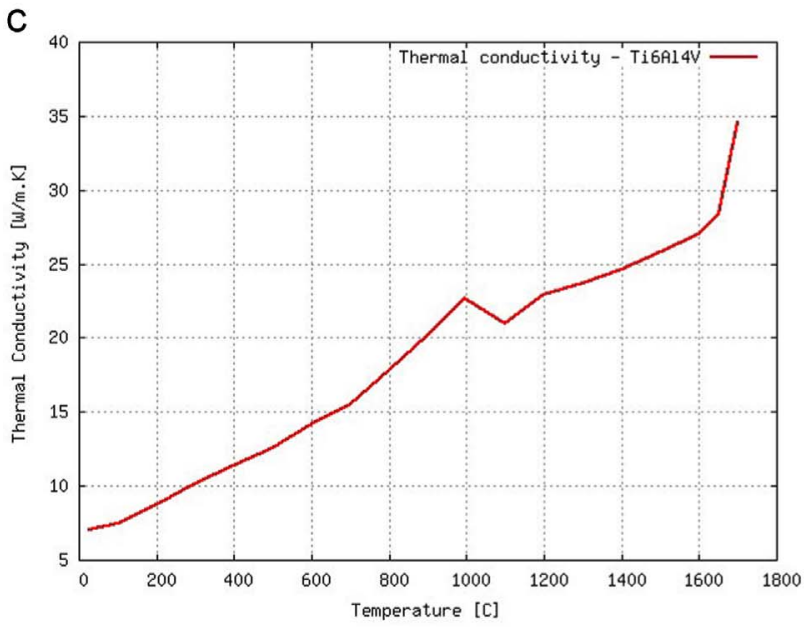

Thermal conductivity

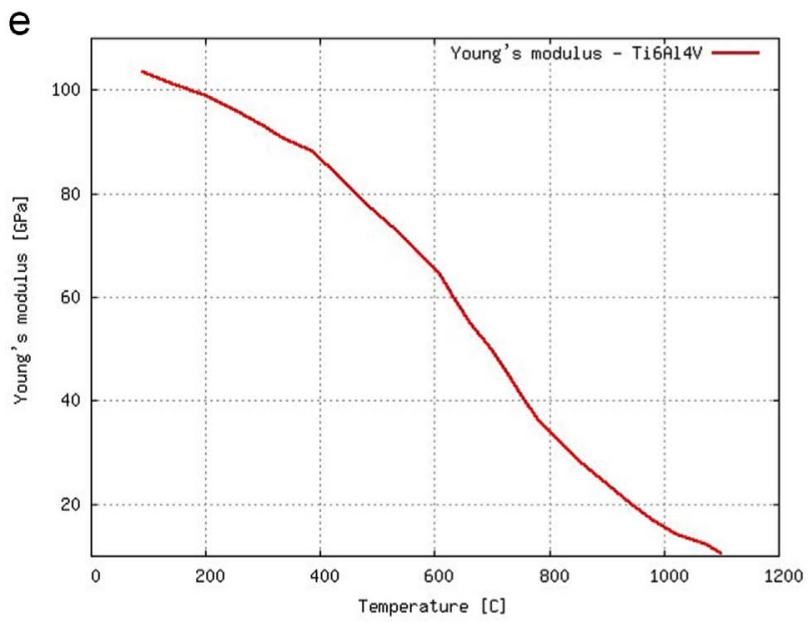

Young's modulus

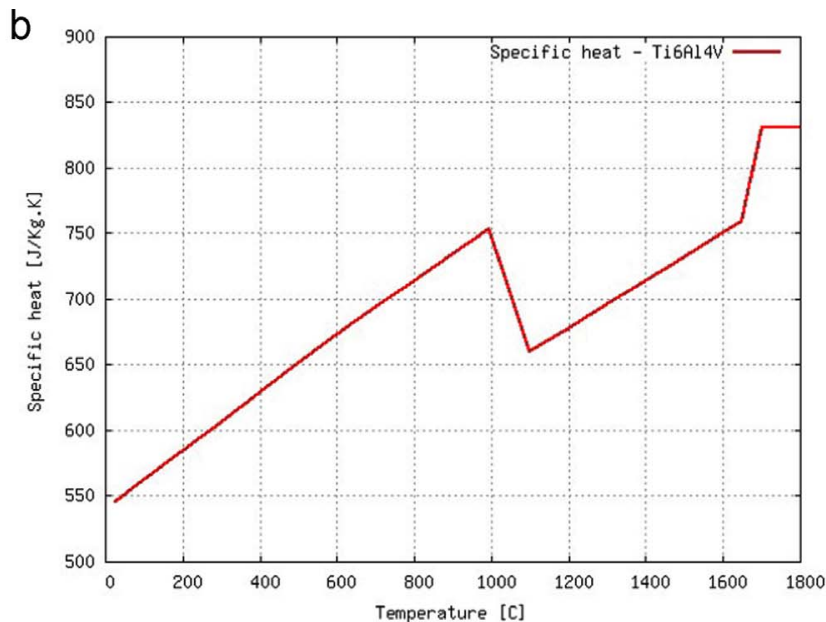

Specific heat

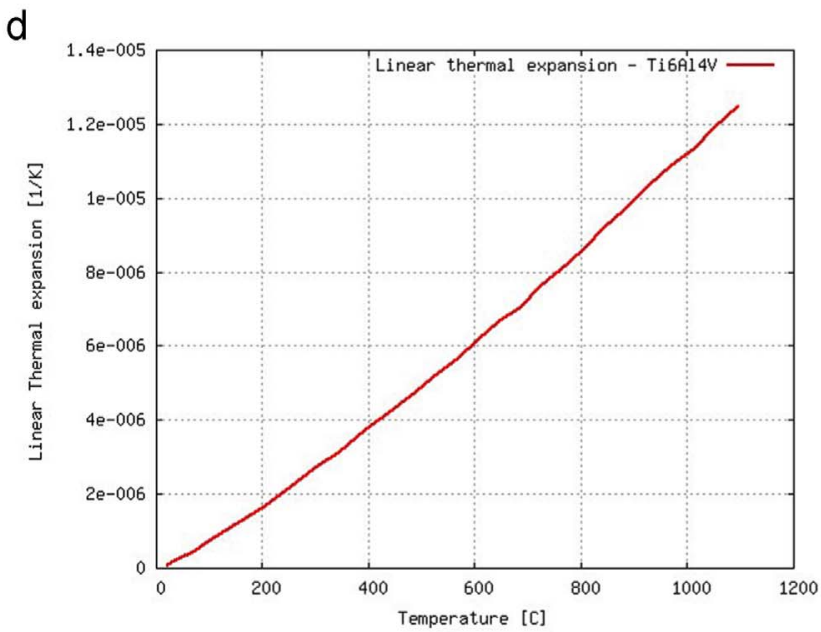

Linear thermal expansion

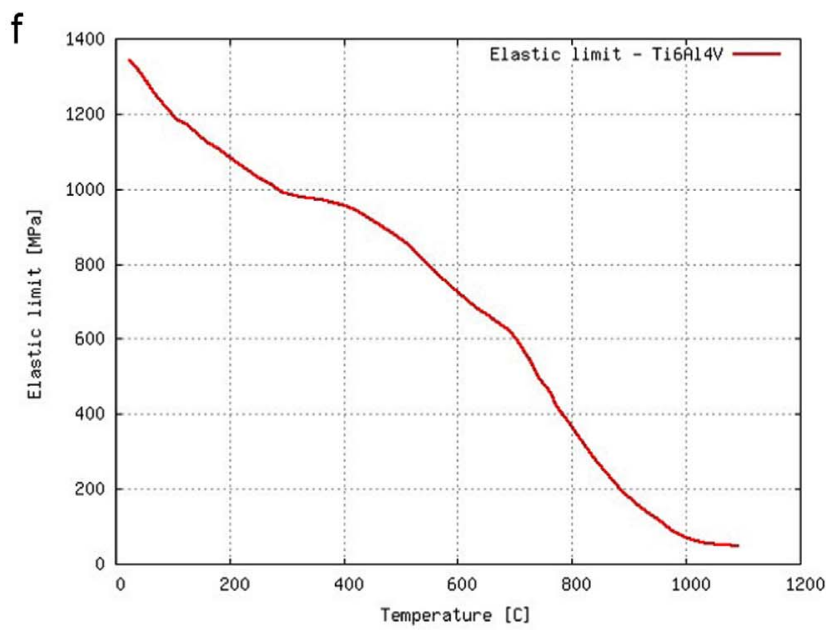

Elastic limit

Fig. 5. Ti6Al4V titanium alloy material properties. 
where $e^{\text {cool }}(T)$ is the thermal dilatation experimented by the material from the initial temperature $T_{o}$ to the current temperature $T$, while $e^{p c}(T)$ is the thermal shrinkage characteristic of the liquid-to-solid phase transformation. The thermal expansion is:

$e^{\text {cool }}(T)=3\left[\alpha(T)\left(T-T_{\text {env }}\right)-\alpha\left(T_{o}\right)\left(T_{o}-T_{\text {env }}\right)\right], \quad\left\{\begin{array}{l}T<T_{S} \\ T>T_{L}\end{array}\right.$

where $\alpha(T)$ is the (temperature dependent) secant thermal expansion coefficient and $T_{\text {env }}$ is the room temperature.

The thermal shrinkage is due to the density variation in the solidification interval $T_{S} \leq T \leq T_{L}$ :

$\frac{d e^{p c}}{d t}=-\frac{1}{\rho} \frac{d \rho}{d t}$

Integrating Eq. (30), it results (see [3]):

$e^{p c}(T)=\ln \frac{\rho_{o}}{\rho(T)} \simeq \frac{\rho_{o}-\rho(T)}{\rho_{o}}, \quad T_{S} \leq T \leq T_{L}$

$\rho_{o}=\rho\left(T_{o}\right)$ being the value of the density at the initial temperature, subjected to the restrictions:

$\rho_{o}=\rho_{S}, \quad T_{o} \leq T_{S}$

$\rho_{o}=\rho_{L}, \quad T_{o} \geq T_{L}$

where $\rho_{S}=\rho\left(T_{S}\right)$ and $\rho_{L}=\rho\left(T_{L}\right)$ are the densities at solidus and liquidus temperatures, respectively.

A (temperature dependent) J2-yield-surface, $\Phi\left(\mathbf{s}, q_{h}, T\right)$, is introduced as:

$\Phi\left(\mathbf{s}, q_{h}, T\right)=\|\mathbf{s}\|-f_{S} R\left(q_{h}, T\right)$

where $R\left(q_{h}, T\right)$ is the (temperature dependent) yield-surface radius

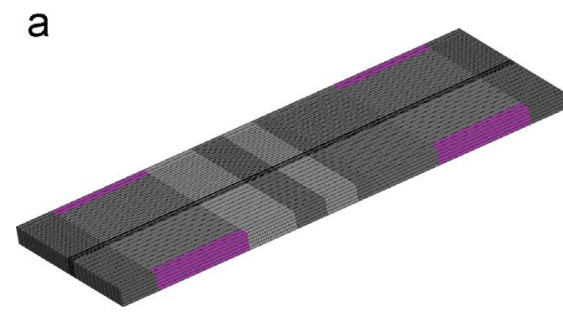

Benchmark 1 defined as:

$R\left(q_{h}, T\right)=\sqrt{\frac{2}{3}}\left[\sigma_{y}(T)-q_{h}\right]$

where $\sigma_{y}(T)$ is the (temperature dependent) initial yield stress (elastic limit) parameter. The stress-like variable, $q_{h}(\xi, T)$, conjugate to the isotropic strain-hardening variable, $\xi$, controls the isotropic hardening phenomena and is defined as:

$q_{h}(\xi, T)=-\left[\sigma_{\infty}(T)-\sigma_{y}(T)\right]\left[1-e^{-\delta(T) \xi}\right]-h(T) \xi$

where $h(T)$ and $\delta(T)$ are the coefficients which control the linear and the exponential isotropic hardening laws, respectively, while $\sigma_{\infty}(T)$ is the (temperature dependent) saturation flow stress parameter.

The visco-plastic strains, $\boldsymbol{\varepsilon}^{v p}=\mathbf{e}^{v p}$, are defined as purely deviatoric. Their definition can be deduced from the principle of maximum plastic dissipation together with the evolution laws of the isotropic strain-hardening variable, $\xi$, as:

$\dot{\mathbf{e}}^{v p}=\dot{\gamma}^{v p} \frac{\partial \Phi\left(\mathbf{s}, q_{h}, T\right)}{\partial \mathbf{S}}=\dot{\gamma}^{v p} \frac{\mathbf{s}}{\|\mathbf{S}\|}=\dot{\gamma}^{v p} \mathbf{n}$

b

Table 1

Welding parameters used for benchmarks 1 and 2 .

\begin{tabular}{ll}
\hline Voltage & $150 \mathrm{kV}$ \\
Current intensity & $100 \mathrm{~mA}$ \\
Speed & $25 \mathrm{~mm} / \mathrm{s}$ \\
Spot size & $1 \mathrm{~mm}$ \\
Penetration & $10 \mathrm{~mm}$ \\
\hline
\end{tabular}

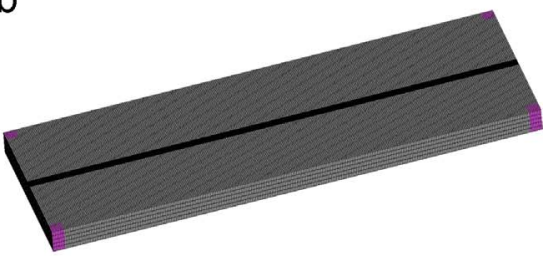

Benchmark 2

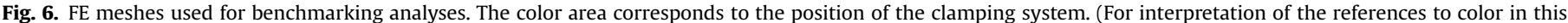
figure caption, the reader is referred to the web version of this paper.)

a

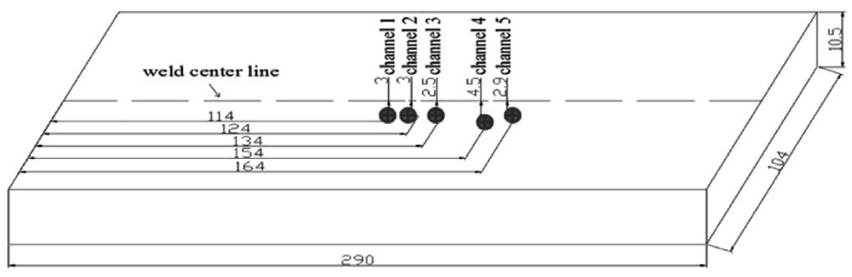

Benchmark 1

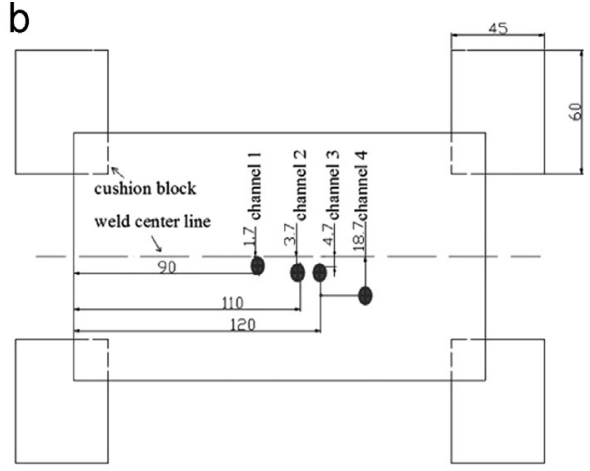

Benchmark 2

Fig. 7. Location of thermocouples for benchmarking analyses. 
a

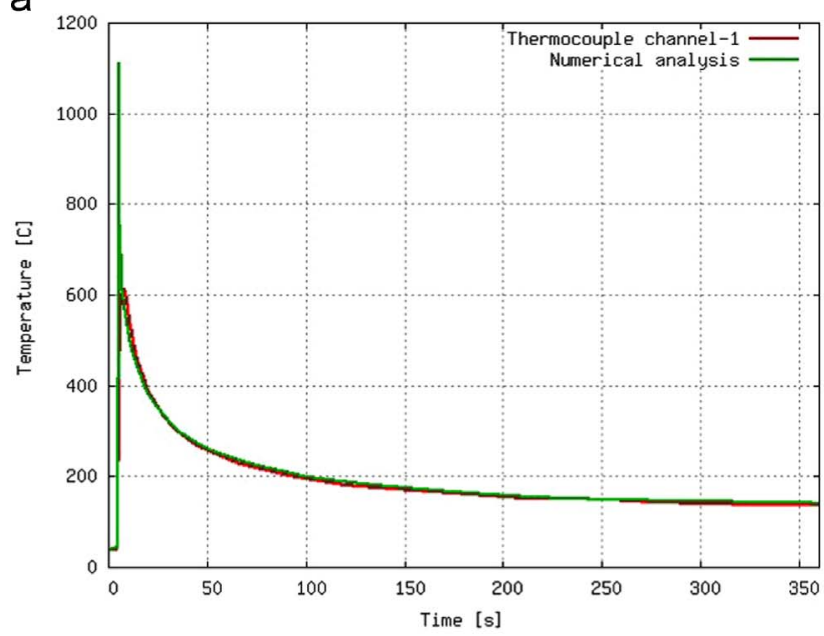

Channel 1

C

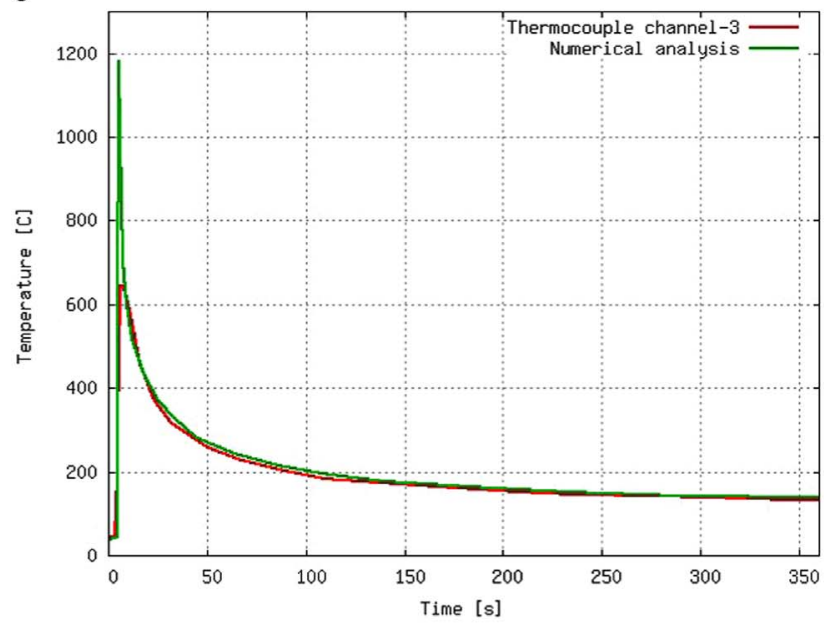

Channel 3 b

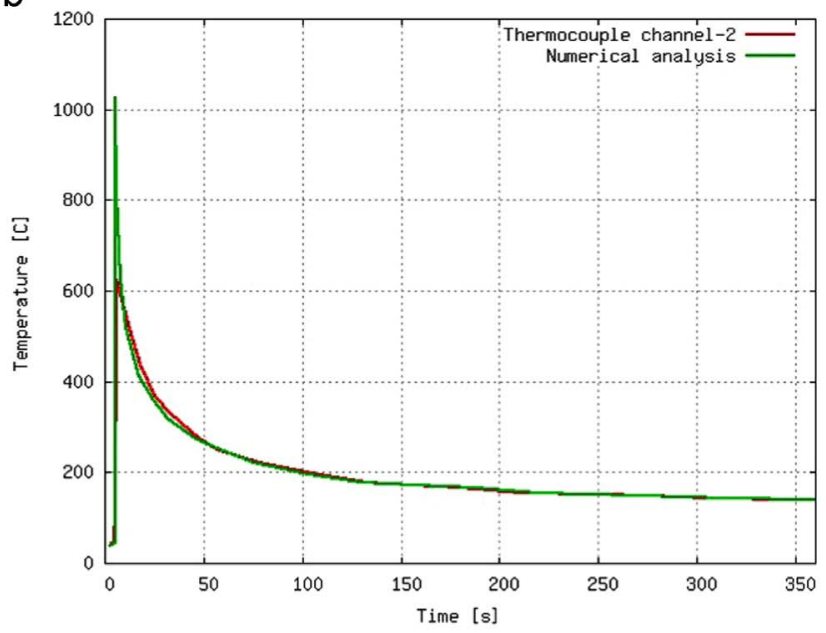

Channel 2

d

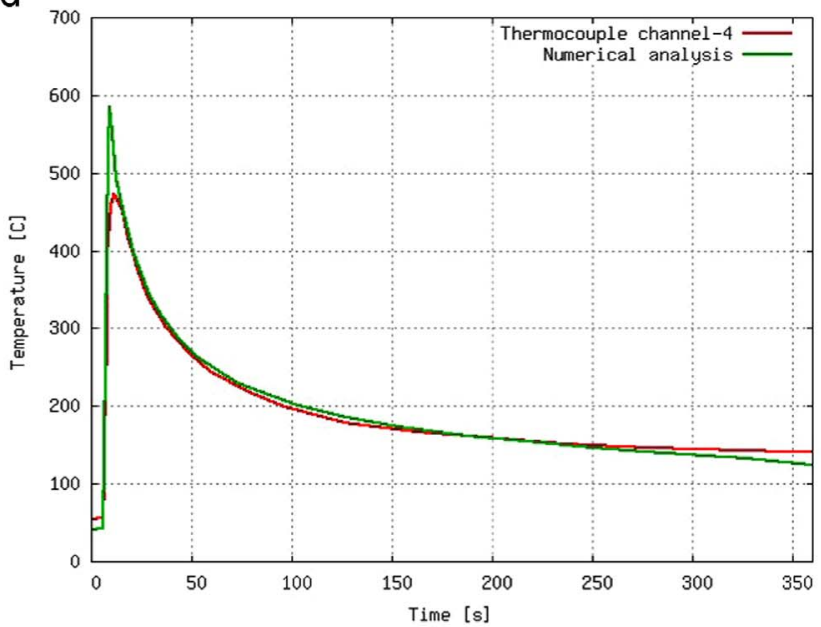

Channel 4

Fig. 8. Temperature evolution at 4 different locations for benchmark 1 . Thermocouple records vs. numerical simulation.

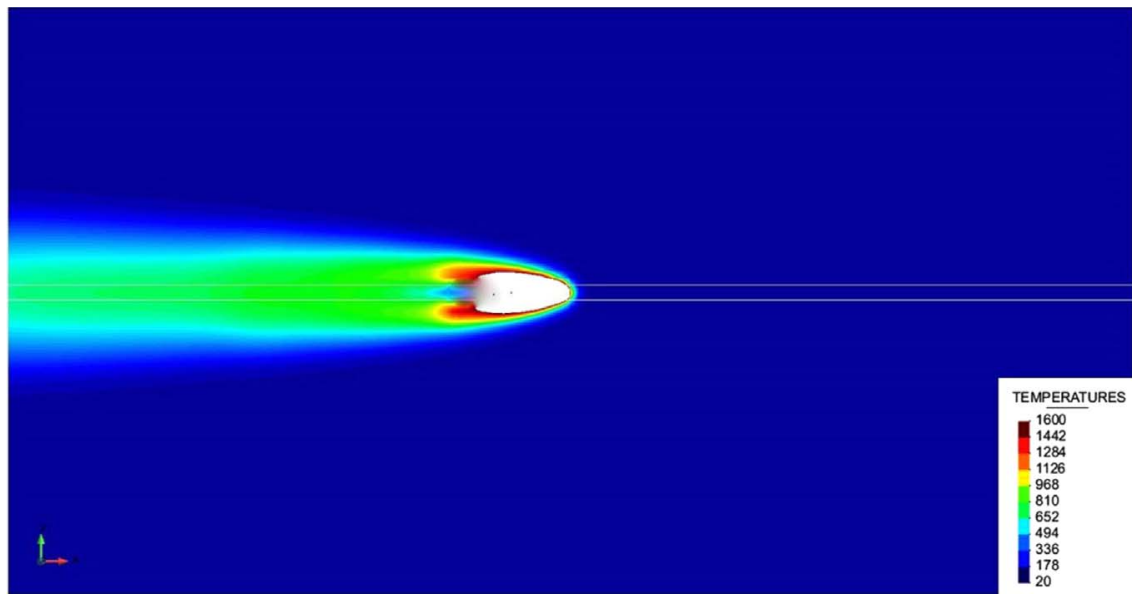

Fig. 9. Temperature contour-fill produced by the EBW process. 
a

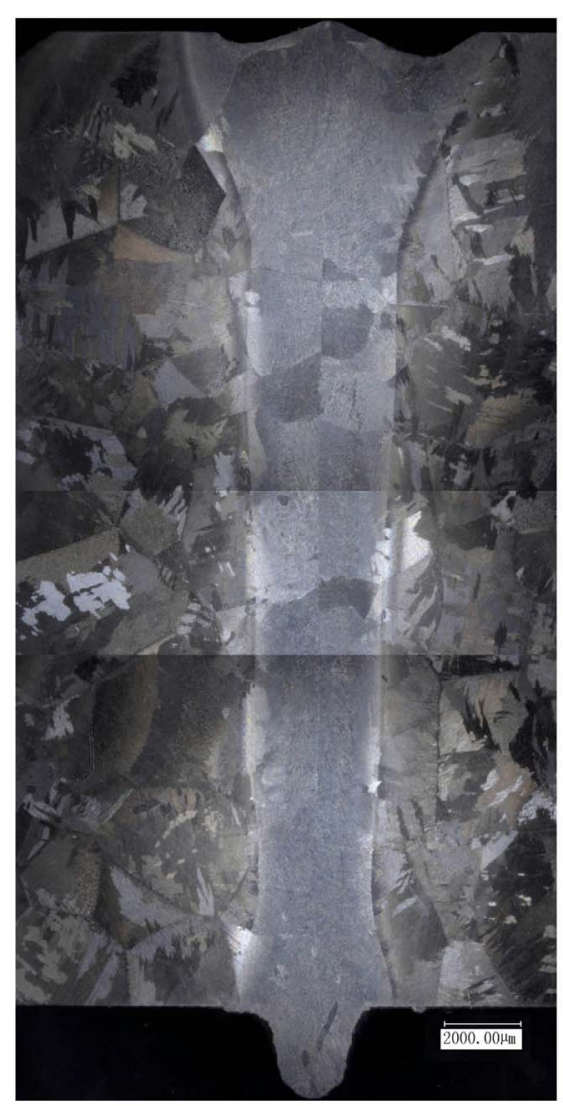

Experimental b

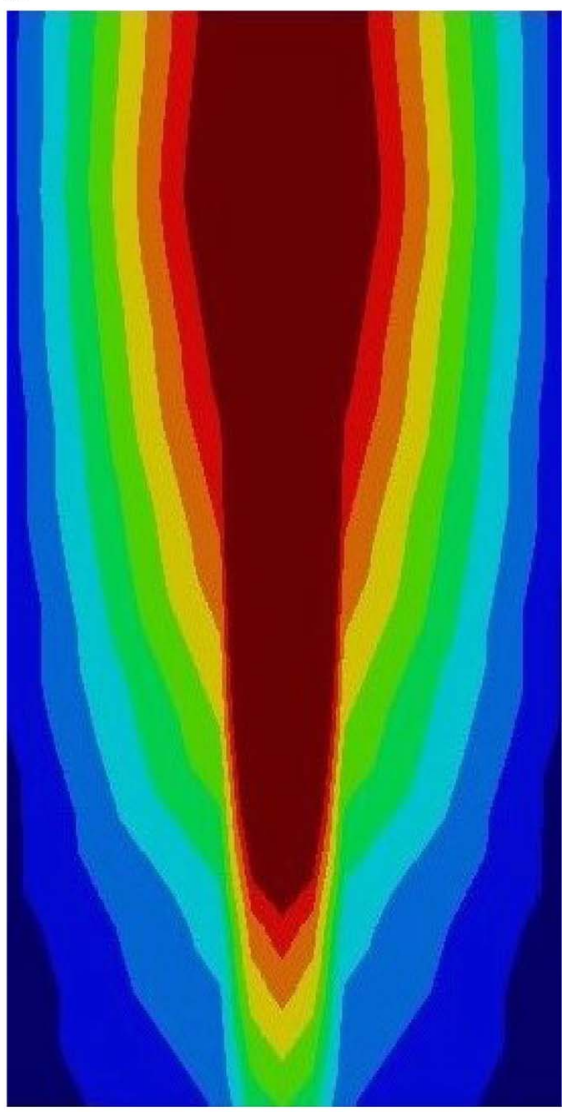

Numerical

Fig. 10. Heat affected zone: experimental evidence vs. numerical simulation.

$\dot{\xi}=\dot{\gamma}^{v p} \frac{\partial \Phi\left(\mathbf{s}, q_{h}, T\right)}{\partial q_{h}}=\dot{\gamma}^{v p} \sqrt{\frac{2}{3}} f_{S}$

where $\mathbf{n}=\frac{\partial \Phi\left(\mathbf{s}, q_{h}, T\right)}{\partial \mathbf{s}}=\frac{\mathbf{s}}{\|\mathbf{s}\|}$ is the normal to the yield surface. The visco-plastic multiplier, $\dot{\gamma}^{v p}$, is computed assuming a rate-dependent evolution law of the form:

$\dot{\gamma}^{v p}=\left\langle\frac{\Phi\left(\mathbf{s}, q_{h}, T\right)}{\eta}\right\rangle^{\frac{1}{m}}$

where $\langle\cdot\rangle$ are the Macaulay brackets. Therefore, when the stress level reaches the yield surface, that is $\Phi\left(s \mathbf{s}, q_{h}, T\right)=0$, then a viscous overstress, $\eta\left(\dot{\gamma}^{v p}\right)^{m}$, is allowed to exceed the yield surface, being $\eta(T)$ the (temperature dependent) plastic viscosity and $m(T)$ its (temperature dependent) rate sensitivity.

The resulting equivalent stress yields:

$\sigma_{e q}=\sqrt{\frac{3}{2}}\left[f_{S} R+\eta\left(\dot{\gamma}^{v p}\right)^{m}\right]$

\subsubsection{Solid phase}

The particularization of the material behavior for the solid phase, $T<T_{S}$ and $f_{S}=1$, is straightforward:

$p=K\left(e^{v o l}-e^{T}\right)$

$\mathbf{s}=2 G\left(\mathbf{e}-\mathbf{e}^{v p}\right)$

where the evolution laws for the visco-plastic strains and the isotropic hardening are deduced from Eqs. (36) and (37) as for the classical J2-thermo-elasto-visco-plastic constitutive model (see $[1,6,12])$.

\subsubsection{Liquid-like phase}

The liquid phase is characterized by a temperature above the liquidus temperature: $T>T_{L}$ and $f_{S}=0$. From Eqs. (23) and (24), both the volumetric and the deviatoric components of the elastic strain tensor vanish. Therefore, on the one hand, Eq. (23) reduces to: $e^{v o l}=e^{T}$. This corresponds to the continuity equation for mass conservation:

$\nabla \cdot \mathbf{u}-e^{T}(T)=0$

On the other hand, Eq. (24) reduces to:

$\mathbf{e}=\mathbf{e}^{v p}$

so that only shear (viscous) deformations occur. The yield-surface radius defined in Eq. (34) reduces as the temperature increases till vanishing $(R=0)$ above the liquidus temperature. Introducing this result into the evolution law of the plastic multiplier (38), a purely viscous model is recovered:

$\mathbf{s}=\eta\left(\dot{\gamma}^{v p}\right)^{m-1} \dot{\mathbf{e}}^{v p}$

as a non-NewtonianNorton-Hoff visco-plastic flow.

Remark 6. Above the melting point, the rate sensitivity of the viscous law is taken as: $m=1$; this means, the Newtonian behavior is assumed.

\subsubsection{Mushy phase}

The material model provides a smooth transition from liquidlike to solid behavior as a function of the solid fraction function, 


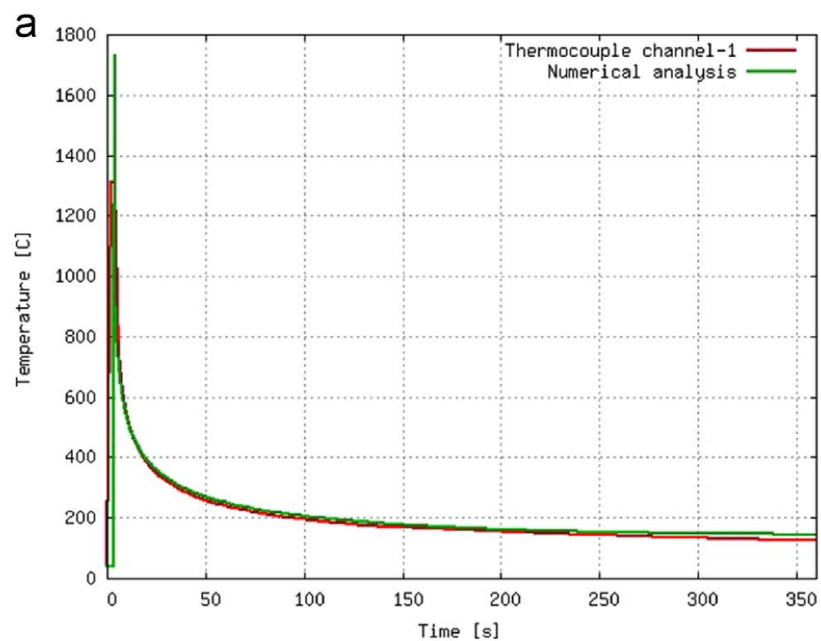

Channel 1

C

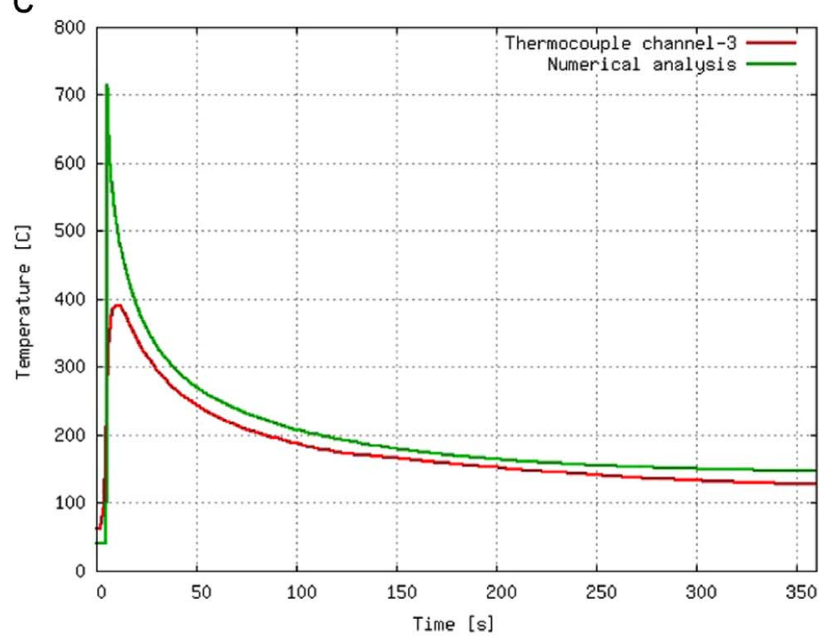

Channel 3

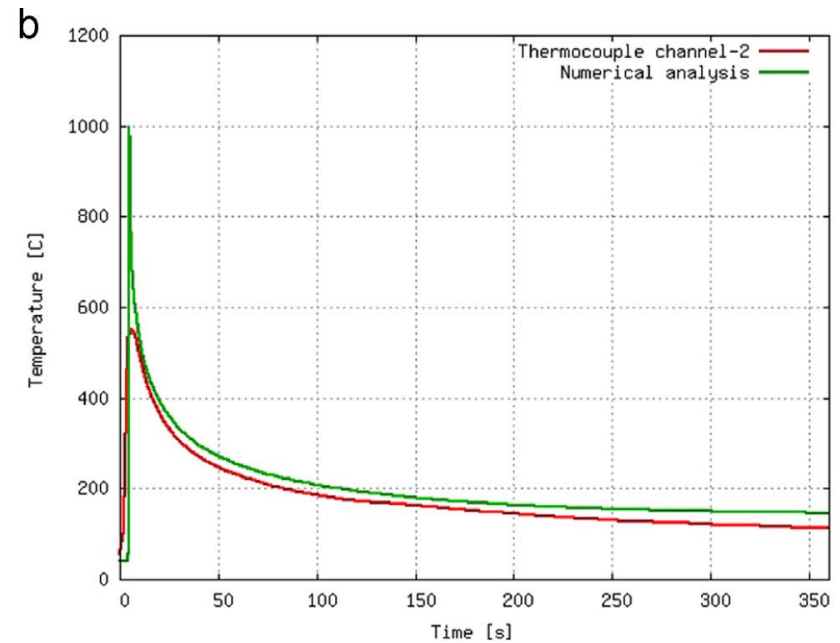

Channel 2

d

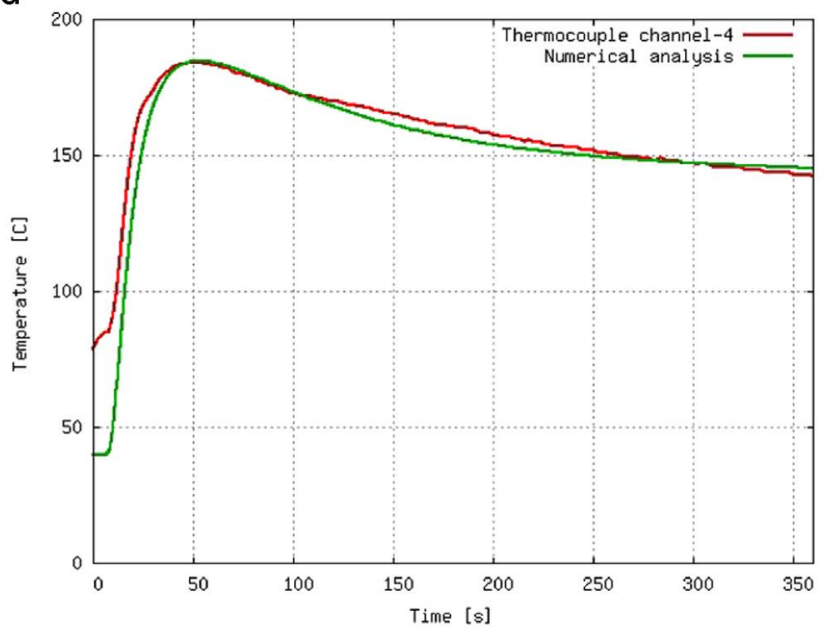

Channel 4

Fig. 11. Temperature evolution at 4 different locations for benchmark 2. Thermocouple records vs. numerical simulation.

$0<f_{S}<1$ in the temperature range $T_{S}<T<T_{L}$. Observe that the yield stress reduces till vanishing in this temperature range. The elastic strains become negligible compared to the visco-plastic deformations. Hence, the mushy phase is actually governed by the visco-plastic flow $[13,19]$.

\subsection{Discrete and stabilized weak form of the mechanical problem}

Within the framework of the standard Galerkin finite element method, the discrete counterpart of the weak form for the mechanical problem (21) is defined by a finite element approximation where both displacement and pressure fields are taken as nodal variables. If continuous equal order linear interpolation $P 1 P 1$ is used to approximate both (discrete) spaces, then the resulting mixed $\mathbf{u} / p$ formulation violates the Ladyzhenskaya-Babuška-Brezzi (LBB) compatibility condition [5]. This produces instabilities in the pressure field and, consequently, poor numerical performance. Stability can be achieved either choosing $\mathbf{u} / p$ interpolation spaces that satisfy the LBB condition (e.g. $P 2 P 1$ elements) or, alternatively, circumventing this condition. A possible choice consists of using a stabilization technique within the framework of Variational Multi Scale (VMS) methods (see the original idea in [24]). In the present work, the Orthogonal Subgrid Scale (OSS) stabilization technique has been adopted to stabilize $P 1 P 1$ mixed $\mathbf{u} / p$ elements with continuous equal order linear interpolations. The OSS technique, originally developed to fulfil the incompressibility condition in CFD problems (see [15]), has been exploited in the solid mechanics context to deal with both elastic incompressibility and J2-plasticity (isochoric) problems (see $[9,8,10,2]$ ).

\section{Experimental set-up}

The EB welding facilities at the Beijing Aeronautical Manufacturing Technology Research Institute (BAMTRI) consist of a high vacuum electron beam welding equipment operating in $85 \mathrm{~m}^{3}$ vacuum chamber. The accelerating voltage of the electron gun is $15 \mathrm{kV}$ with a nominal power of $60 \mathrm{~kW}$. Fig. 3a shows the EB welding facilities at BAMTRI.

The welding process has been carried out in accordance with AIRBUS quality standards (AIP01-04-011-Specification for Electron Beam Welding of Hard Metals). The joint quality has been inspected by $X$-ray to discard pieces with internal cracks or excessive porosity, meeting A-Class quality standards. 
a

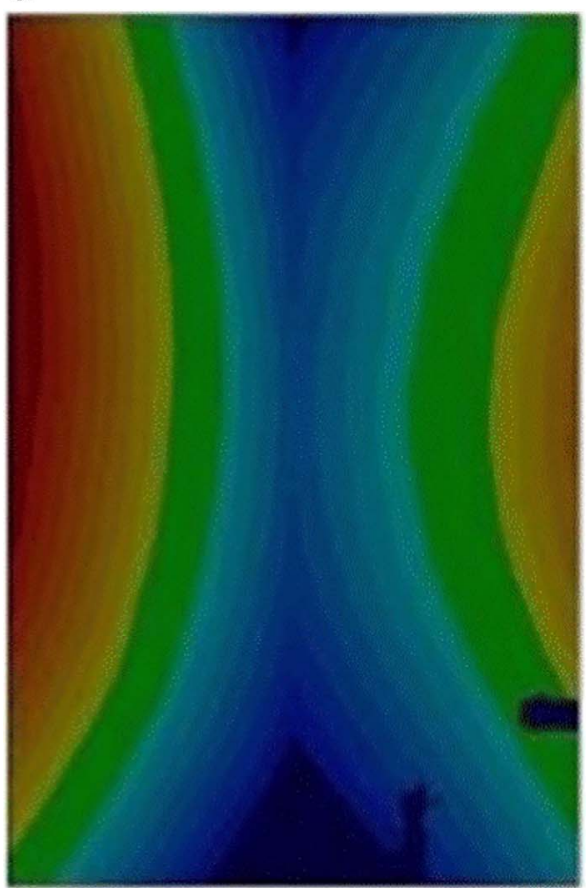

Experimental b

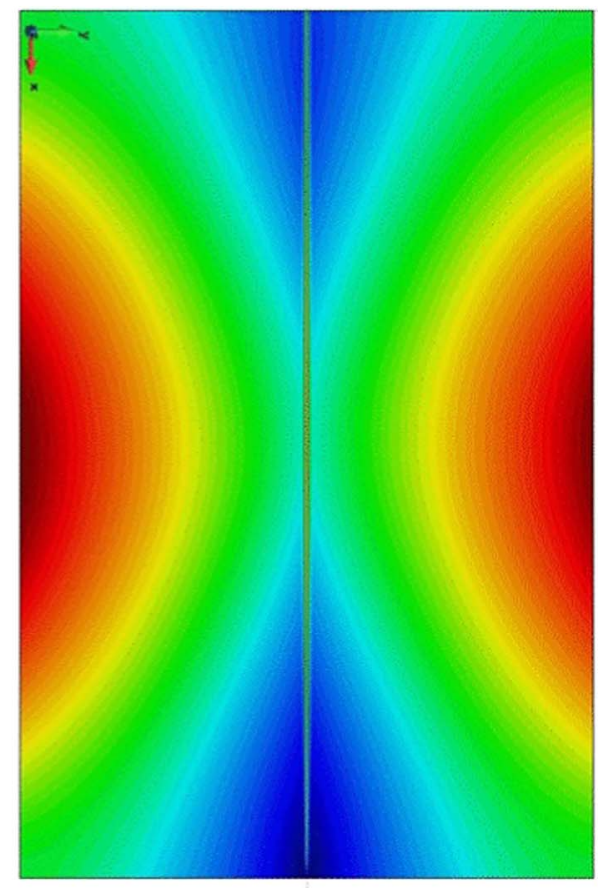

Numerical

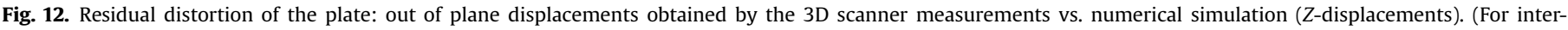
pretation of the references to color in this figure caption, the reader is referred to the web version of this paper.)

Temperature measurements have been carried out by thermocouples located at different distances from the welding line avoiding the radiation flux induced by the power source. All measurements have been duplicated (from both sides of the welding line) to ensure quality control. Fig. 4a shows a welding sample with the corresponding thermocouples for the temperature measurements during the whole duration of the welding process.

Konica-Minolta [range-7] is the 3D laser scanning system used to measure the distortions of the different samples after cooling at room temperature (see Fig. 3b). The measuring method consists of a triangulation light block technique, which uses a semiconductor laser with a wave-length of $660 \mathrm{~nm}$, a resolution of 1.31 megapixels $(1280 \times 1024)$ and a tolerance of $4 \mu \mathrm{m}$. The measurement process starts by marking a grid of reference points on each surface of the body. Later, the laser source is used for a threedimensional scanning of the body surfaces using a narrow light plane (less than $0.4 \mathrm{~mm}$ wide) identifying the position of the reference points with very high accuracy (see Fig. 4b). The measurements are imported into a CAD system to compare the reference geometry with the deformed one and color-filled contour plot can be produced and easily compared with the results coming from the numerical simulation.

Numerical simulation of the EBW process requires the characterization of the material properties to be used for the analysis according to the constitutive model available. Their values depend on the temperature, covering the full range from room temperature to fusion temperature $\left(1655^{\circ} \mathrm{C}\right)$.

In the present work, the target material is Ti6Al4V titanium alloy. Fig. 5 shows the temperature-dependent properties used for this alloy within the available temperature range $[4,20]$.

Poisson's ratio used is $\nu=0.342$. The liquidus-to-solidus phasechange is defined by a latent heat of $L=290 \mathrm{~kJ} / \mathrm{kg}$, together with the liquidus and solidus temperatures of $1655^{\circ} \mathrm{C}$ and $1605^{\circ} \mathrm{C}$, respectively.

\section{Numerical results}

This section describes the assessment of the in-house coupled thermo-mechanical FE software developed for the numerical simulation of the EBW process [7].

The results obtained in the simulation analyses are presented in terms of temperature graphs at different points (virtual thermocouples) of the structure, as well as contour-fills of both temperature and distortion distributions using the in-house pre-postprocessor GiD [22]. These results are compared with the corresponding measurements obtained at BAMTRI research labs.

Two analyses have been carried out: firstly, two benchmarks are used to calibrate and validate the software in terms of thermal (temperature evolution) and mechanical (residual distortions) response, respectively. The two samples are very similar a part from the boundary conditions (clamping system) and the location of the thermocouples. They consist of rectangular titanium plates joined together using a constant EB power source. Next, an industrial case is presented. Two different welding configurations are tested on a door frame component from AIRBUS to assess their industrial feasibility.

\subsection{Calibration benchmarking}

The first example consists of a Titanium (Ti6Al4V) plate, $290 \mathrm{~mm}$ long, $104 \mathrm{~mm}$ wide and $10.5 \mathrm{~mm}$ thick. Fig. 6a shows the FE mesh used to perform the numerical simulation. It consists of 13.846 nodes and 16.314 hexahedral elements. A finer mesh has been used close to the welding line to be able to capture the high temperature gradient induced by the EBW process. To capture the mechanical distortion accurately, 5 elements have been placed through the thickness of the plate. Mixed $\mathbf{u} / p$ FE technology has been used to deal with the isochoric plastic strains induced by the J2-plastic model adopted as well as for incompressible behavior of the fluid-like phase. 
The colored zones in Fig. 6a represent the surfaces in contact with the clamping system which have been used to restrain the movement of the plates. Conduction heat transfer at the contact interface between the plate and the clamping system is considered. The heat transfer coefficient used for Newton's model is $500 \mathrm{~W} / \mathrm{m}^{2} \mathrm{~K}$.

The software uses an automatic time-stepping algorithm which moves the heat source element-by-element (Courant's number: $C u=1)$. This allows for most accurate results but, at the same time, is the more CPU-time consuming solution.

a

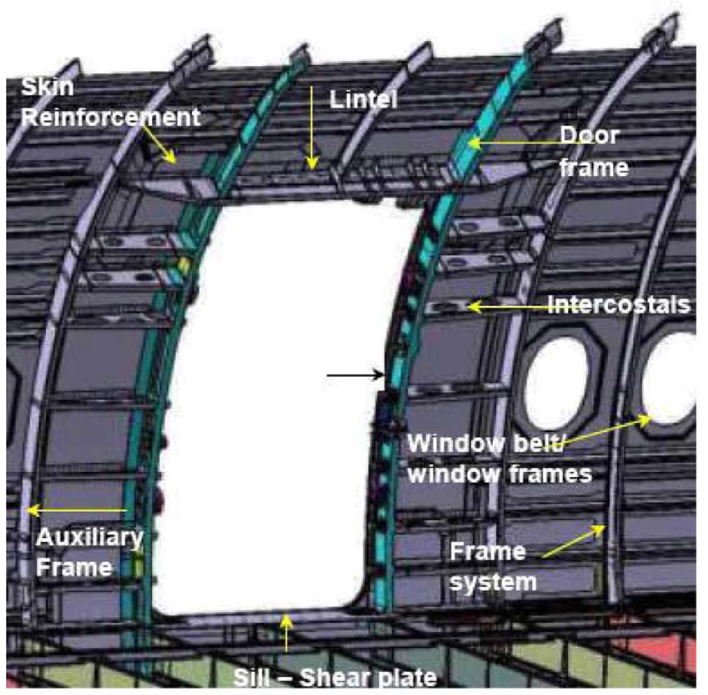

Door frame structure

C

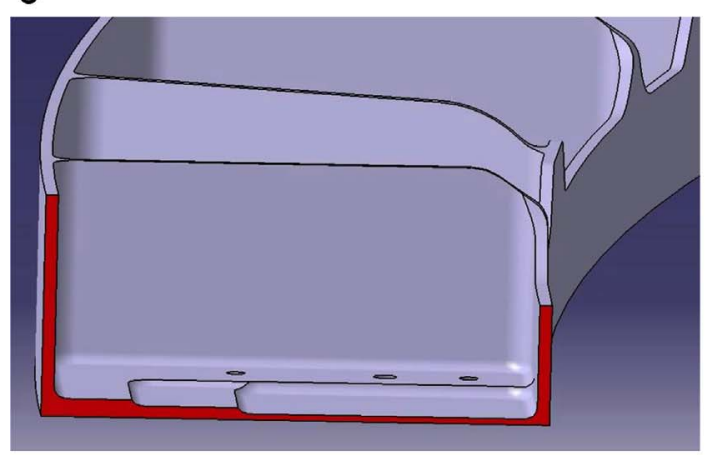

Component cross-section
Table 2

Process parameters used for welding AIRBUS door frame component. Option 1: variable thickness without the flange insert.

\begin{tabular}{|c|c|c|c|}
\hline & $2 \mathrm{~mm}$ & $4 \mathrm{~mm}$ & $6 \mathrm{~mm}$ \\
\hline Voltage $(\mathrm{kV})$ & 150 & 150 & 150 \\
\hline Current intensity (mA) & 20 & 40 & 60 \\
\hline Speed $(\mathrm{mm} / \mathrm{s})$ & 25 & 25 & 25 \\
\hline Spot size $(\mathrm{mm})$ & 1 & 1 & 1 \\
\hline Penetration (mm) & 2 & 4 & 6 \\
\hline
\end{tabular}

b

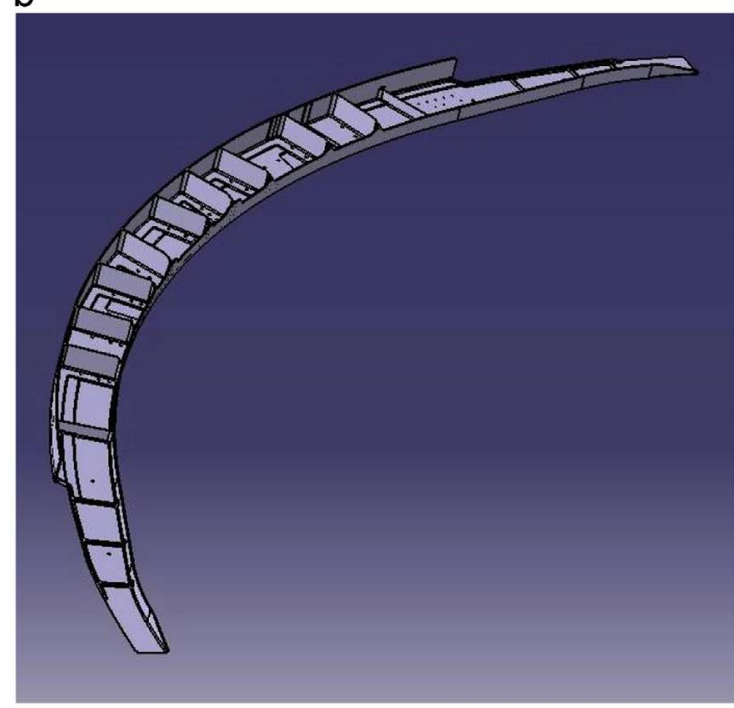

Selected component

d

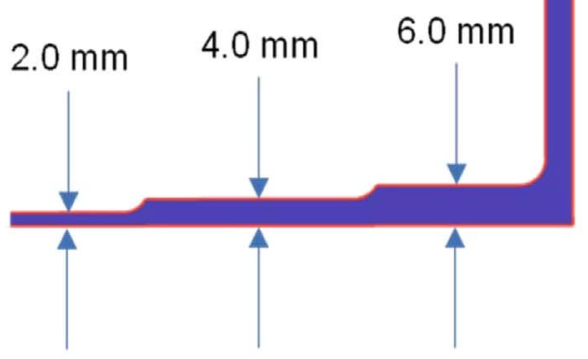

Thicknesses of 2,4 and 6 [mm].

Fig. 13. AIRBUS door frame component.

a

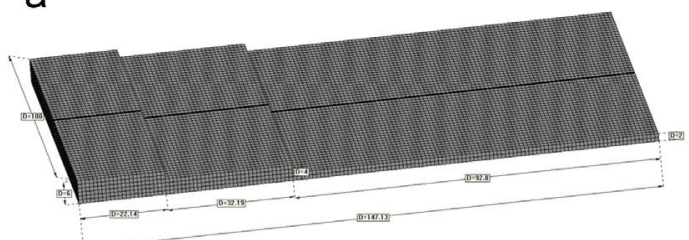

$i \rightarrow x$ b

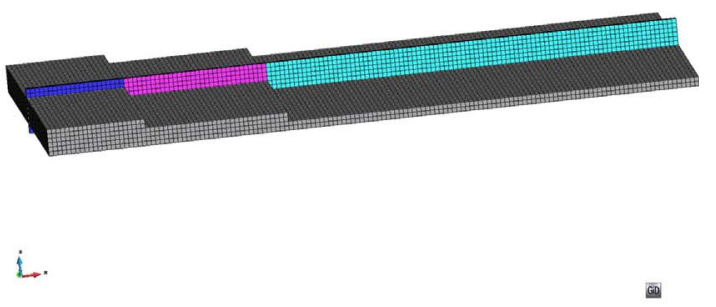

Without flange

Using flange insert

Fig. 14. FE meshes of door frame component. 


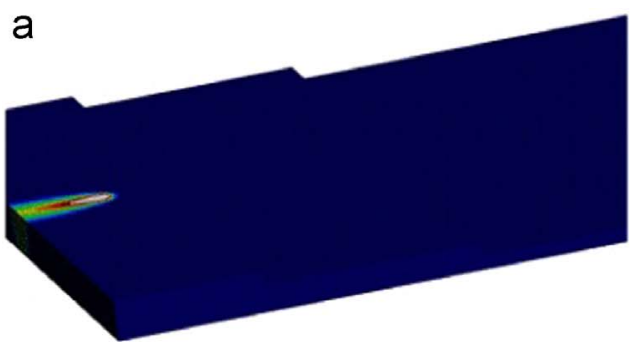

b

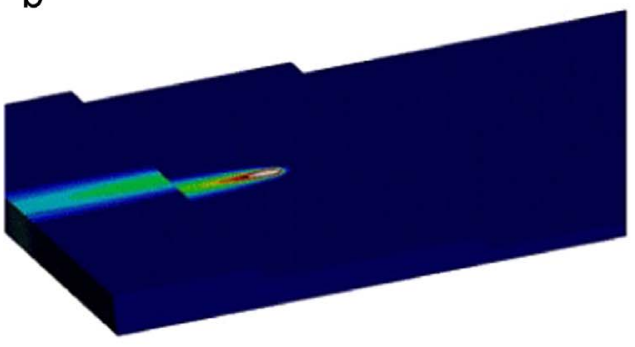

C

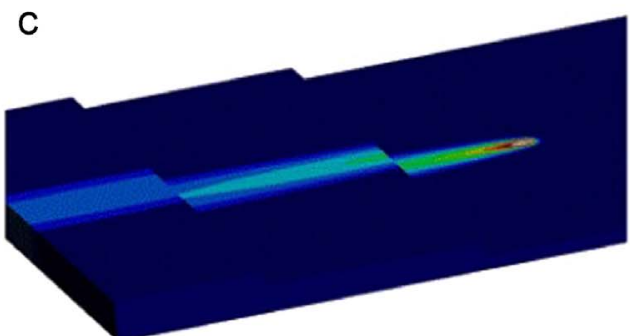

d

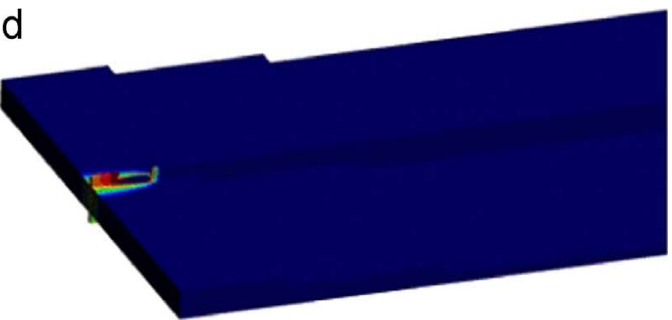

e

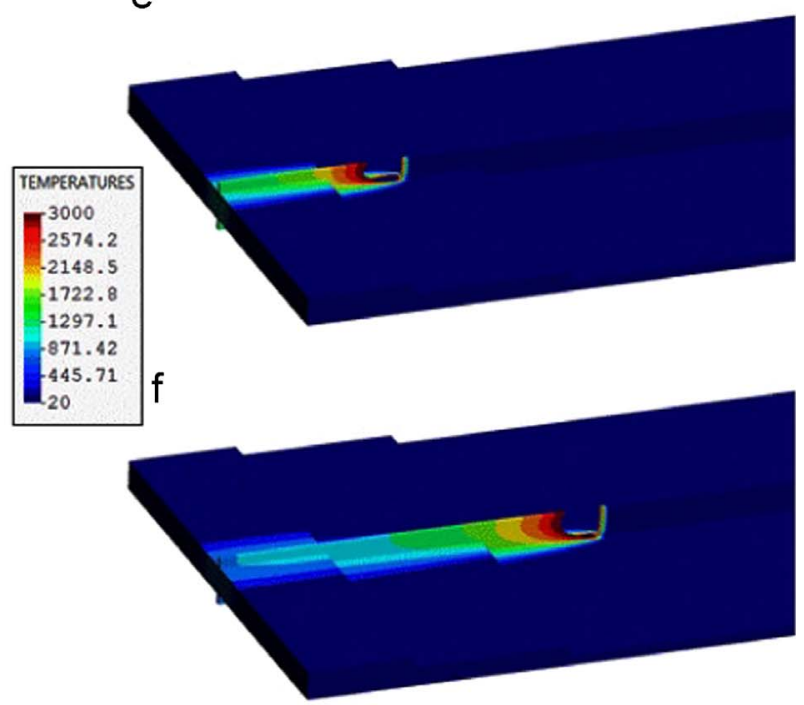

Fig. 15. EBW process through the cross section of AIRBUS door frame component. Temperature contour-fill: without flange (left) vs. using flange insert (right).

a

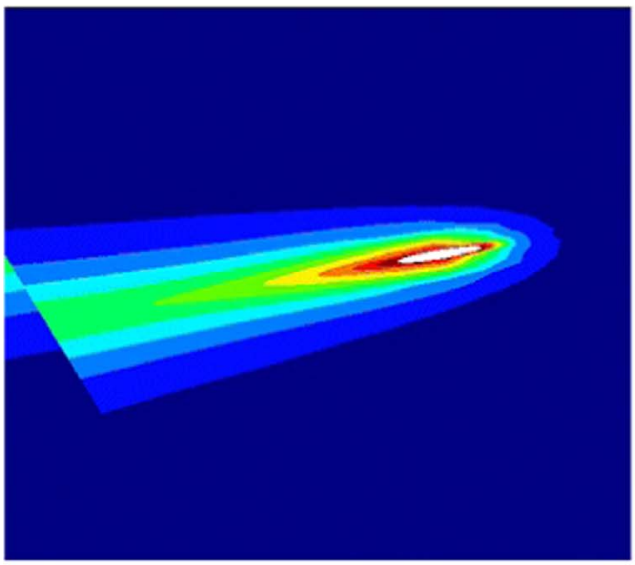

Without flange insert b
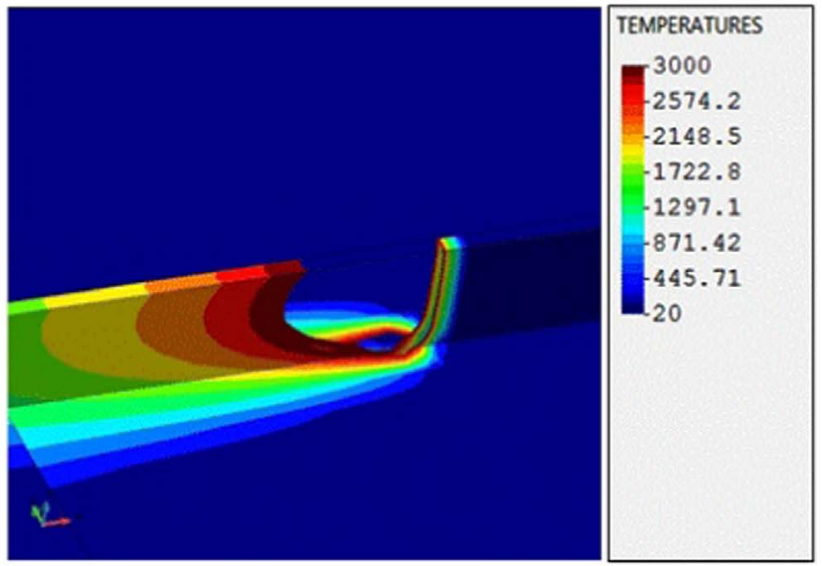

Using flange insert

Fig. 16. Detail of the temperature contour-fill showing the molten-pool for the 2 welding configurations analyzed.

The EBW process is performed in a vacuum chamber so that the heat flux by convection can be neglected and the heat absorbed by the plate during the EB welding process can be evacuated by radiation heat flux only. The emissivity coefficient used for the radiation model is 0.8 .

Fig. 7a shows the location of the 5 thermocouples used for the measurement of the temperature evolution during the EBW process. They are placed at 3, 3, 2.5, 4.5 and $2.9 \mathrm{~mm}$ from the welding line, respectively.

Table 1 shows the welding parameters used for the experimental setting. The same parameters have been assumed in the numerical simulation to define the power source.
Fig. 8a-d compares the thermal measurements obtained by the thermocouples 1-4 with the corresponding numerical results obtained. There is good agreement between the corresponding temperature graphs at all the thermocouples locations. The only noticeable difference is in the peak temperature. This difference depends on the thermal inertia of the thermocouples used for the temperature recording. The smaller and the more conductive the thermocouples are, the faster are the temperature measurements. Observe that the difference in the peak temperature reduces when the location is far from the welding line.

Fig. 9 shows the typical temperature contour-fill during the EBW process simulation. It is possible to appreciate that the heat 
source is very much focused, affecting only the material nearby the welding line. The key-hole shape is also shown. Temperatures in the key-hole are much higher than the fusion temperature and here the material is vaporized. Fig. 10a shows the resulting microstructure after welding and it is possible to appreciate the size and shape of the HAZ. Fig. 10b shows the corresponding HAZ obtained through numerical simulation. Remarkable agreement is obtained.

A second welding is performed on a similar sample and it is used for a double-check to prove the previous measurements. The same plate size, material and welding process parameters have been used as for the previous case. The main difference consists of the position of the clamping system, affecting the mechanical response. The position of the clamping system that is the surfaces used to prescribe the vertical movement are detailed in Fig. 6b (color area). The FE mesh used for the numerical analysis is identical to the previous one.

Fig. $7 \mathrm{~b}$ shows the location of the 4 thermocouples used for the measurement of the temperature evolution during the EBW process. They are placed at 1.7, 3.7, 4.7 and $18.7 \mathrm{~mm}$ from the welding line, respectively.

The comparison between the thermal measurements and the corresponding numerical curves is shown in Fig. 11a-d. Once again the agreement is noticeable.

Fig. 12a shows the final distortions after cooling. The experimental contour-fill is obtained interpolating the out of plane ;displacements recorded by the 3D scanning system. The corresponding numerical result is shown in Fig. 12b in terms of $z$-displacement contour-fill. The same color-scale has been used for both results. The comparison shows a good agreement from both qualitative and quantitative points of view. On the one hand, the result obtained through the numerical simulation presents perfect symmetry with respect to the welding line because the clamping prescriptions are enforced exactly. On the other hand, the experimental result is very sensitive to the effectiveness of the clamping system and it is clear that this is not able to prevent the movement of the plate due to the thermal dilatation induced by the welding process.

\subsection{AIRBUS door frame component}

The selected component is a large-scale complex thin-walled titanium structure. This component is part of the AIRBUS door frame as shown in Fig. 13a. Fig. 13b shows the component once isolated from the assembled structure which forms the door frame. The welding analysis is performed through the crosssection shown in Fig. 13c. The key point is that it exists a variable thickness (2, 4 and $6 \mathrm{~mm}$, respectively), making the welding operation more demanding (see Fig. 13d).

Two different welding configurations are analyzed:
1. EBW process using a variable power source according to the variable thickness of the section to be welded.

2. EBW process using a constant power source during the whole welding operation. This process configuration is feasible by inserting a consumable flange along the welding line. The EB penetration and spot size correspond to the flange height and thickness, respectively.

The first analysis corresponds to the first option, which assumes a variable power source according to the thickness of the titanium plate to be welded. The airframe structure has been simplified removing the two lateral flanges. However, both length and thickness of each section of the plate have been maintained as in the original design of the airframe component. Fig. 14a shows the geometry proposed for the welding analysis and the corresponding FE mesh made of 24.084 elements and 33.000 nodes.

The component is made of titanium (Ti6Al4V) and the material properties are the same as for the benchmarks. Only heat radiation loss has been assumed: the emissivity coefficient used is 0.8 with an environment temperature of $20^{\circ} \mathrm{C}$.

The power source parameters used for each thickness have been obtained scaling the current intensity in Table 1 . The welding parameters used for the analysis are shown in Table 2. An efficiency of $75 \%$ is assumed to account for the actual power absorption.

In the second analysis, a $10 \mathrm{~mm}$ high and $1 \mathrm{~mm}$ thick flange has been inserted along the welding line. Fig. 14b shows the geometry with the welding flange inserted between the two parts to be jointed, and the corresponding FE discretization using 28.180 elements and 38.140 nodes.

The welding process parameters are given in Table 1 and they are the same used to calibrate the model in the benchmarking analyses. In fact, now the EB penetrates through the full thickness of the flange ( $10 \mathrm{~mm}$ as for the benchmarking samples) independently of the actual thickness of the plates to be welded. Also in this cases, an efficiency of $75 \%$ has been assumed.

Fig. 15 shows on the left-hand side 3 contour-fills which correspond to 3 different time-steps of the first analysis (variable power source), during the welding process of the 6,4 and $2 \mathrm{~mm}$ thickness plates, respectively. On the right-hand side the corresponding contour-fills for the flanged option. In both cases (variable and constant power source), it is possible to produce high quality weld independently of the plate thickness to be welded. The size and shape of the molten-pool is very similar for both options (see Fig. 16) without appreciable differences along the welding line as well as in the HAZ.

Fig. 17 shows the location of two thermocouples close to the welding line for both welding configurations. The first thermocouple falls inside the HAZ ( $2 \mathrm{~mm}$ from the welding line), while a

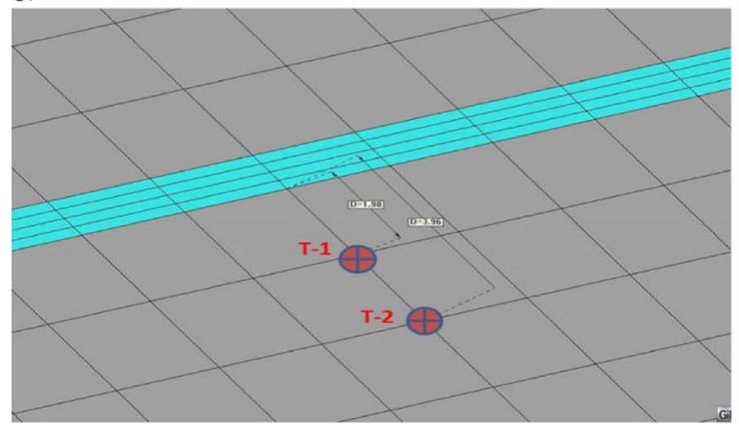

Without flange $\mathrm{b}$

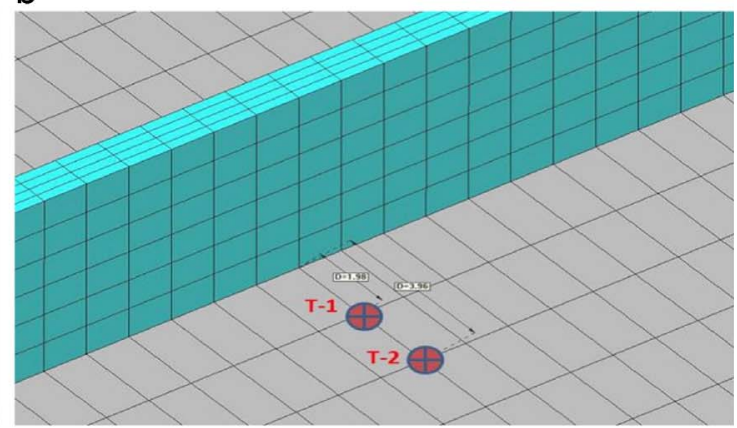

Using flange insert

Fig. 17. Thermocouple location. 


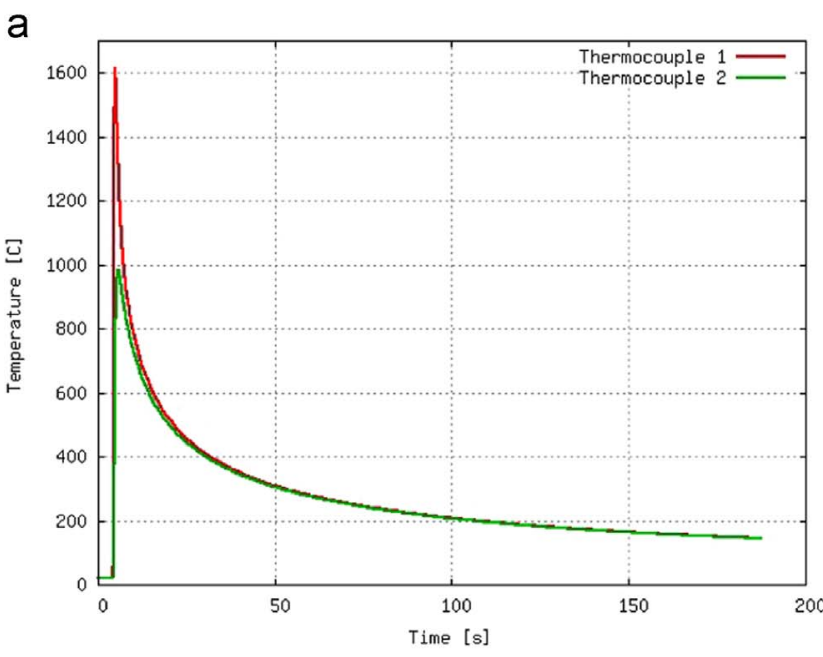

Without flange b

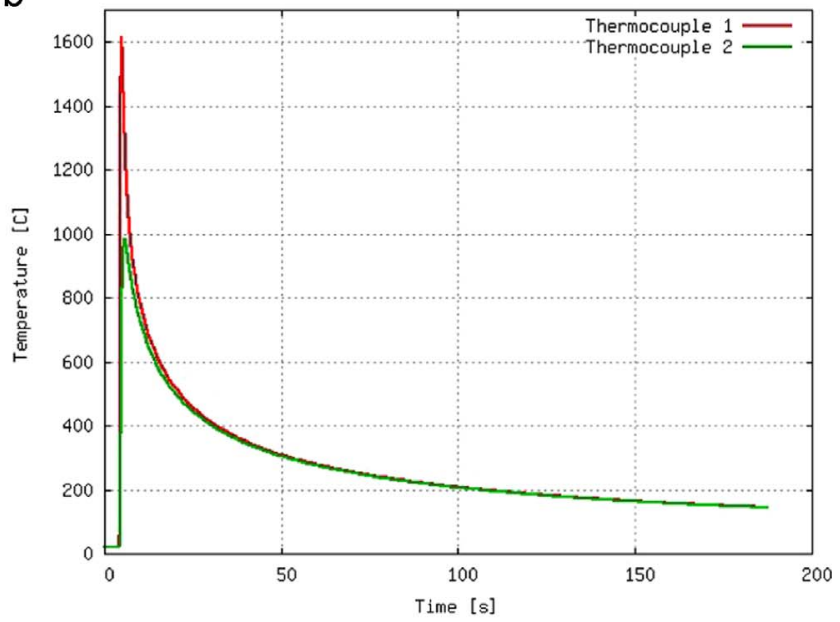

Using flange insert

Fig. 18. Temperature evolution at 2 different thermocouple locations. Experimental measurements vs. numerical analysis.

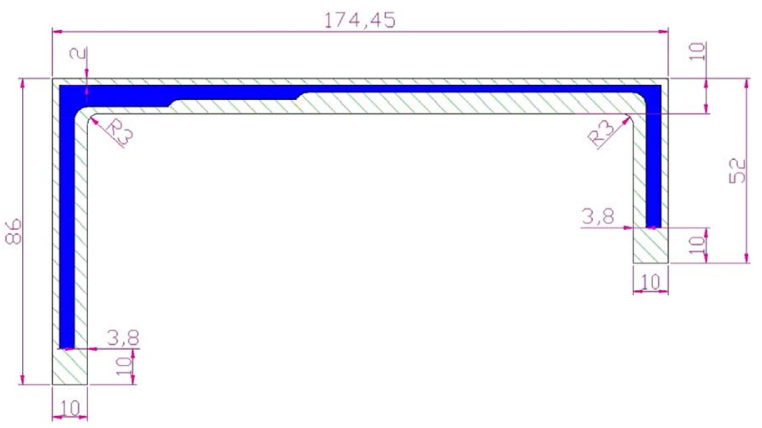

Fig. 19. Welding configuration adopted by AIRBUS to weld the cross-section of the door frame component.

the second one is placed nearby $(4 \mathrm{~mm})$. The corresponding temperature histories are presented in Fig. 18. The peak values as well as the cooling times are very similar for the two welding configurations.

From the industrial point of view, this result is very encouraging, particularly when adopting the second configuration. In fact, this option allows for either complex welding shapes or sections with a variable thickness. The use of a (suitable) constant power source simplifies the welding operation and the preliminary power source calibration. Fig. 19 shows the variable thickness section of the AIRBUS door frame component. A constant height flange (10 mm high and $1 \mathrm{~mm}$ thick) is inserted along the entire section to be welded allowing for a constant EB power source.

\section{Conclusion}

In this work, a FE framework for the numerical simulation of the EBW process is presented. Apropos constitutive behavior within the entire temperature range from room temperature to the fusion temperature is detailed. A precise description of the EB power source is introduced at continuum level together with the corresponding corrections due to the FE discretization and timestepping scheme.

The numerical model has been calibrated and validated through an experimental campaign carried out at BAMTRI research labs. Experimental and numerical temperature profiles as well as residual out-of-plane displacements are compared with noticeable agreement. The application to an industrial case has proved the capability of the proposed numerical tool to validate suitable welding configurations for demanding shapes.

\section{Acknowledgments}

Financial support from the EC - Collaborative Program under the COLTS Project - Casting of Large Ti Structures - within the 7th Framework Programme and funding from the Spanish Ministry of Economy and Competitivity under the EACY project (Ref. MAT2013-48624-C2-1-P) - Enhanced accuracy computational and experimental framework for strain localization and failure mechanisms - within the Excellency Programme for Knowledge Generation are gratefully acknowledged.

This research is supported by State Administration of Foreign Experts Affairs of China through the High-end Experts Recruitment Program.

\section{References}

[1] C. Agelet de Saracibar, M. Cervera, M. Chiumenti, On the formulation of coupled thermoplastic problems with phase-change, Int. J. Plast. 15 (1999) 1-34.

[2] C. Agelet de Saracibar, M. Chiumenti, Q. Valverde, M. Cervera, on the orthogonal subgrid scale pressure stabilization of small and finite deformation j2 plasticity, Comput. Methods Appl. Mech. Eng. 195 (2006) 1224-1251.

[3] M. Bellet, B-G. Thomas, Solidification Macroprocesses (Modeling of stress, distortion and hot tearing), In: J.R. Groza, J.F. Shackelford, E.J. Lavernia, M.T Powers (Eds.), Materials Processing Handbook, Chapter 27, CRC Press, Taylor and Francis, Florida, 2007.

[4] M.J. Bermingham, S.D. McDonald, M.S. Dargusch, D.H. StJohn, Microstucture of cast titanium alloys, Mater. Forum 31 (2007).

[5] F. Brezzi, M. Fortin, Mixed and Hybrid Finite Element Methods, Springer, New York, 1991.

[6] M. Cervera, C. Agelet de Saracibar, M. Chiumenti, Thermo-mechanical analysis of industrial solidification processes, Int. J Numer. Methods Eng. 46 (1999) $1575-1591$.

[7] M. Cervera, C. Agelet de Saracibar, M. Chiumenti, COMET: COupled MEchanical and Thermal analysis, Data Input Manual, Version 5.0, Technical Report IT-308, 2002, 〈http://www.cimne.com/comet〉.

[8] M. Cervera, M. Chiumenti, Q. Valverde, C. Agelet de Saracibar, Mixed linear/ linear simplicial elements for incompressible elasticity and plasticity, Comput. Methods Appl. Mech. Eng. 192 (2003) 5249-5263.

[9] M. Chiumenti, Q. Valverde, C. Agelet de Saracibar, M. Cervera, A stabilized formulation for incompressible elasticity using linear displacement and pressure interpolations, Comput. Methods Appl. Mech. Eng. 191 (2002) $5253-5264$. 
[10] M. Chiumenti, Q. Valverde, C. Agelet de Saracibar, M. Cervera, A stabilized formulation for incompressible plasticity using linear triangles and tetrahedra, Int. J. Plast. 20 (2004) 1487-1504.

[11] M. Chiumenti, C. Agelet de Saracibar, M. Cervera, On the numerical modelling of the thermo-mechanical contact for metal casting analysis, J. Heat Transf. 130 (2008) 1-10.

[12] M. Chiumenti, M. Cervera, A. Salmi, C. Agelet de Saracibar, N. Dialami, K. Matsui, Finite element modeling of multi-pass welding and shaped metal deposition processes, Comput. Methods. Appl. Mech. Eng. 199 (2010) 2343-2359, http://dx.doi.org/10.1016/j.cma.2010.02.018.

[13] M. Chiumenti, M. Cervera, C. Agelet de Saracibar, N. Dialami, Numerical modeling of frictional stir welding processes, Comput. Methods. Appl. Mech. Eng. 254 (2013) 353-369.

[14] M. Chiumenti, X. Lin, M. Cervera, W. Lei, Y. Zheng, W. Huang, Numerical simulation and experimental calibration of Additive Manufacturing by blown powder technology. Part I: thermal analysis, Rapid Prototyp. J. (2016) http:// dx.doi.org/10.1108/RPJ-10-2015-0136 (in press).

[15] R. Codina, Stabilization of incompressibility and convection through orthogonal sub-scales in finite element methods, Comput. Methods. Appl. Mech. Eng. 190 (2000) 1579-1599.

[16] D.B. Darmadi, Validating the accuracy of heat source model via temperature histories and temperature field in bead-on-plate welding, Int. J. Eng. Technol. 11 (5) (2011) 111505-116868.

[17] D. Deng, H. Murakawa, Numerical simulation of temperature field and residual stress in multi-pass welds in stainless steel pipe and comparison with experimental measurements, Comput. Mater. Sci. 37 (2006) 269-277.

[18] D. Deng, H. Murakawa, W. Liang, Numerical simulation of welding distortion in large structures, Comput. Methods Appl. Mech. Eng. 196 (2007) 4613-4627.

[19] N. Dialami, M. Chiumenti, M. Cervera, C. Agelet de Saracibar, An apropos kinematic framework for the numerical modelling of friction stir welding, Comput. Struct. 117 (2013) 48-57.

[20] J.W. Elmer, T.A. Palmer, S.S. Babu, W. Zhang, T. DebRoy, Phase transformation dynamics during welding of Ti-6Al-4V, J. Appl. Phys. 12 (2004) 95, http://dx. doi.org/10.1063/1.1737476.
[21] S.S. Gajapathi, S.K. Mitra, P.F. Mendez, Controlling heat transfer in micro electron beam welding using volumetric heating, Int. J. Heat Mass Transf. 54 (2011) 5545-5553.

[22] GiD: The Personal Pre and Post Preprocessor (http://www.gidhome.com).

[23] J. Goldak, A. Chakravarti, M. Bibby, A new finite element model for welding heat sources, Metall. Trans. 15B (1984) 299-305.

[24] T.J.R. Hughes, Multiscale phenomena: green's function, Dirichlet-to Neumann formulation, subgrid scale models, bubbles and the origins of stabilized formulations, Comput. Methods Appl. Mech. Eng. 127 (1995) 387-401.

[25] P. Lacki, K. Adamus, Numerical simulation of the electron beam welding process, Comput. Struct. 89 (2011) 977-985.

[26] L.-E. Lindgren, H. Runnemalm, M. Näsström, Simulation of multipass welding of a thick plate, Int. J. Numer. Methods Eng. 44 (1999) 1301-1316.

[27] L.-E. Lindgren, Finite element modelling of welding Part 1: increased complexity, J. Thermal Stress. 24 (2001) 141-192.

[28] L.-E. Lindgren, Finite element modelling of welding Part 2: improved material modelling, J. Thermal Stress. 24 (2001) 195-231.

[29] L.-E. Lindgren, Finite element modelling of welding. Part 3: efficiency and integration, J. Thermal Stress. 24 (2001) 305-334.

[30] D.W. Lobitz, J.D. Mc Clure, R.E. Nickell, Residual stresses and distorsions in multi pass welding, in: Proceedings of the ASME W AM, Numerical Modelling of Manufacturing Processes, PVP-PB-25, 1977, pp. 1-18.

[31] M. Rahman, W. Maurer, W. Ernst, R. Rauch, N. Enzinger, Calculation of hardness distribution in the HAZ of micro-alloyed steel, Wel World 58 (2014) 763-770, http://dx.doi.org/10.1007/s40194-014-0156-5.

[32] D. Rosenthal, Mathematical theory of heat distribution during welding and cutting, Welding J. 20 (5) (1941) 220-234.

[33] Y. Tian, C. Wang, D. Zhu, Y. Zhou, Finite element modeling of electron bean welding of a large complex Al alloy structure by parallel computations, J. Mater. Process. Technol. 199 (2008) 41-48.

[34] B. Zhang, T. Wang, X. Duan, G. Chen, J. Feng, Temperature and stress fields in electron beam welded Ti-15-3 alloy to 304 stainless steel joint with copper interlayer sheet, Trans. Nonferrous Met. Soc. China 22 (2012) 398-403. 